TRANSACTIONS OF THE

AMERICAN MATHEMATICAL SOCIETY

Volume 182, August 1973

\title{
ON RATIONALLY CONVEX HULLS
}

\author{
BY \\ RICHARD F. BASENER $\left({ }^{1}\right)$
}

ABSTRACT. For a compact set $X \subseteq \mathbf{C}^{n}$, let $h_{r}(X)$ denote the rationally convex hull of $X$; let $\Delta$ denote the closed unit disk in $C_{\text {; }}$ and, following Wermer, for a compact set $S$ such that $\partial \Delta \subseteq S \subseteq \Delta$ let $X_{S}=S \times S \cap \partial^{2}$. It is shown that

$$
h_{r}\left(X_{S}\right)=\left\{(z, w) \in S \times S \mid u_{S}(z)+u_{S}(w) \leq 1\right\}
$$

where $u_{S}$ is a function on $S$ which, in the case when $S$ is smoothly bounded, is specified by requiring $\left.u_{S}\right|_{\partial \Delta}=0,\left.u_{S}\right|_{\partial S \backslash \partial \Delta}=1$ and $\left.u_{S}\right|_{\text {int } S}$ harmonic. In particular this provides a precise description of $h_{r}(X)$ for certain sets $X \subseteq \mathbf{C}^{2}$ with the property that $h_{r}(X) \neq X$, but $h_{r}(X)$ does not contain analytic structure (as Wermer demonstrated, there are $S$ for which $X=X_{S}$ has these properties). Furthermore, it follows that whenever $h_{r}\left(X_{s}\right) \neq X_{s}$ then there is a Gleason part of $h_{r}\left(X_{S}\right)$ for the algebra $R\left(X_{S}\right)$ with positive four-dimensional measure. In fact, the Gleason part of any point $(z, w) \in h_{r}\left(X_{S}\right) \cap$ int $\Delta^{2}$ such that $u_{s}(z)+$ $u_{s}(w)<1$ has positive four-dimensional measure.

A similar idea is then used to construct a compact rationally convex set $Y \subseteq \mathrm{C}^{2}$ such that each point of $Y$ is a peak point for $R(Y)$ even though $R(Y) \neq C(Y)$; namely, $Y=\widetilde{X}_{T}=\left\{(z, w) \in \mathrm{C}^{2}|z \in T| w \mid,=\sqrt{1-|z|^{2}}\right\}$ where $T$ is any compact subset of int $\Delta$ having the property that $R(T) \neq C(T)$ even though there are no nontrivial Jensen measures for $R(T)$. This example is more concrete than the original example of such a uniform algebra which was discovered by Cole. It is possible to show, for instance, that $R\left(\widetilde{X}_{T}\right)$ is not even in general locally dense in $C\left(\widetilde{X}_{T}\right)$, a possibility which had been suggested by Stuart Sidney.

Finally, smooth examples (3-spheres in $\mathbf{C}^{6}$ ) with the same pathological properties are obtained from $X_{S}$ and $\tilde{X}_{T}$.

Introduction. Let $X$ be a compact subset of $\mathbf{C}^{n} \cdot R_{0}(X)$ is the algebra of all

Received by the editors November 8, 1972.

AMS (MOS) subject classifications (1970). Primary 32E99, 46J 10.

Key words and phrases. Rationally convex hull, analytic structure, peak point.

(1) This paper contains the results of the author's Ph.D. thesis written at Brown University under the direction of John Wermer, to whom the author is deeply grateful. This research was supported in part by the National Science Foundation under Grant GP-28574. 
rational functions $P / Q$ on $\mathbf{C}^{n}$ with $P, Q$ polynomials and $Q \neq 0$ on $X, R(X)$ is the uniform closure of $R_{0}(X)$ in $C(X) . b_{r}(X)$, the rationally convex hull of $X$, is the set $\left\{z \in \mathbf{C}^{n} \mid\right.$ for all polynomials $P$ such that $P(z)=0, P$ has a zero on $\left.X\right\}$.

While every $X \subseteq \mathrm{C}$ is rationally convex, i.e., $b_{r}(X)=X$, in $\mathrm{C}^{2}$ even a compact arc may fail to be rationally convex. (See the example of Wermer [1] and Rudin [2] or [3, p. 70]. Wermer's example is in $\mathrm{C}^{3}$.) The rationally convex hull of a general "circled" compact set in $\mathrm{C}^{n}$ has been described very thoroughly by de Leeuw [4], [5] and Rossi [6]. A set $X \subseteq \mathrm{C}^{n}$ is "circled" if, whenever $\left(z_{1}, \cdots\right.$, $\left.z_{n}\right) \in X,\left(\lambda_{1}, \cdots, \lambda_{n}\right) \in \mathbf{C}^{n}$ and $\left|\lambda_{j}\right|=1$ for $1 \leq j \leq n$, it follows that $\left(\lambda_{1} z_{1}, \cdots\right.$, $\left.\lambda_{n} z_{n}\right) \in X$. Suppose that $X$ is a "circled" compact set in $\mathbf{C}^{n}$, that int $X$ is a dense connected subset of $X$, and that int $X$ meets every coordinate axis $\left\{z_{j}=0\right\}$ which $X$ meets. In this case their result is that

$h_{r}(X)=$ closure $\left\{\left(z_{1}, \ldots, z_{n}\right) \in \mathbb{C}^{n} \mid\left(\log \left|z_{1}\right|, \ldots, \log \left|z_{n}\right|\right)\right.$

$\epsilon$ linear convex hull $\left.\left\{\left(s_{1}, \ldots, s_{n}\right) \in \mathbf{R}^{n} \mid\left(e^{s_{1}}, \ldots, e^{s_{n}}\right) \in X\right\}\right\}$.

The general case follows by taking a decreasing intersection of such sets (see also [3, pp. 71-76]).

In the first section of this paper we describe $h_{r}(X)$ for certain sets $X$ contained in the boundary of the bicylinder which were first discussed by Wermer [7]. Given a compact set $S$ contained in the closed unit disk $\Delta$ and containing the boundary of the disk $\partial \Delta$, let

$$
X_{S}=\left\{(z, w) \in \mathbf{C}^{2}|| z \mid=1 \text { and } w \in S \text {, or }|w|=1 \text { and } z \in S\right\} \text {. }
$$

A set $Y \subseteq \mathbf{C}^{n}$ is said to "contain analytic structure" if there is a nonconstant analytic mapping from a disk to $\mathrm{C}^{n}$ whose image is contained in $Y$. As was observed by Soltzenberg [8], the open mapping theorem implies that if $Y$ contains analytic structure, then one of the coordinate projections $z_{i}(Y)$ has nonempty interior in $\mathbf{C}$. Wermer in [7] constructed an $S$ such that $b_{r}\left(X_{S}\right) \backslash X_{S} \neq \varnothing$ but int $S=$ $\varnothing$, whence $b_{r}\left(X_{S}\right) \subseteq S \times S$ does not contain analytic structure. (The first example of this general type is due to Stolzenberg [8], who proved the existence of a compact set $X \subseteq \mathrm{C}^{2}$ which is not polynomially convex but whose polynomially convex hull does not contain analytic structure.)

First assume that $S$ is a compact connected subset of $\Delta$ containing $\partial \Delta$, and write $S=\bigcap_{n=1}^{\infty} S_{n}$ where

(i) each $S_{n}$ is a compact connected smoothly bounded subset of $\Delta$;

(ii) $S_{n}$ is a neighborhood of $S_{n+1}$ in $\Delta$.

Define $u_{n} \in C\left(S_{n}\right)$ by requiring that $u_{n}$ be harmonic on int $S_{n}, u_{n} \equiv 0$ on $\partial \Delta$ 
and $u_{n} \equiv 1$ on $\partial S_{n} \backslash \partial \Delta$. For $z \in S$ let

$$
u_{S}(z)=\lim _{n \rightarrow \infty} u_{n}(z)
$$

The main result of $\$ 1$ may then be stated as

Theorem 1. $b_{r}\left(X_{S}\right)=\left\{(z, w) \in S \times S \mid u_{S}(z)+u_{S}(w) \leq 1\right\}$.

The determination of $b_{r}\left(X_{S}\right)$ yields some quantitative information:

Theorem 2. If $(z, w) \in h_{r}\left(X_{S}\right) \backslash X_{S}$ and $u_{S}(z)+u_{S}(w)<1$, then the Gleason part of $(z, w)$ in $R\left(X_{S}\right)$ bas positive 4-dimensional measure.

This leads almost at once to

Corollary. If $h_{r}\left(X_{S}\right) \neq X_{S}$, then the 4-dimensional measure of $b_{r}\left(X_{S}\right) \backslash X_{S}$ is positive.

One consequence of Theorem 1 is that there are compact connected subsets $S$ of $\Delta$ which contain $\partial \Delta$ and for which $X_{S}$ is rationally convex even though $R\left(X_{S}\right) \neq C\left(X_{S}\right)$. For example, let $S$ be a compact connected subset of $\Delta$ such that $S \supseteq \partial \Delta, R(S) \neq C(S)$, but the only Jensen measures for $R(S)$ are point masses (such an $S$ can be obtained by a simple modification of an example due to Browder (see $\left[9\right.$, p. 192])). It can be shown that $u_{S} \equiv 1$ on $S \backslash \partial \Delta$, so that $b_{r}\left(X_{S}\right)=X_{S}$ by Theorem 1. This suggests that such $S$ might yield interesting rationally convex sets in other ways.

The second section of this paper is essentially independent of the first. In it we exhibit a concrete example of a uniform algebra $A$ defined on a compact metric space (its maximal ideal space) with the properties:

(I) $A$ does not contain all continuous functions on its maximal ideal space;

(II) every point of the maximal ideal space of $A$ is a peak point for $A$;

(III) $A$ is finitely generated.

The first example of such an algebra is due to Cole (unpublished); his example is a modification of an algebra satisfying (I) and (II) which is constructed in his thesis ([10]; or see [9, pp. 255-262]).

Let $T$ be any compact subset of int $\Delta$ such that the only Jensen measures for $R(T)$ are trivial, but $R(T) \nRightarrow C(T)$; Cole's construction also begins with such a $T$ (examples of such $T$, besides the previously mentioned one in [9], are found in [11] and [12]). We use $T$ to obtain a compact subset of the boundary of the unit ball in $\mathrm{C}^{2}$ by defining 


$$
\widetilde{X}_{T}=\left\{\left.(z, w) \in \mathbf{C}^{2}|| z\right|^{2}+|w|^{2}=1, z \in T\right\}
$$

Theorem 3. If $A=R\left(\tilde{X}_{T}\right)$, then conditions (I), (II) and (III) are satisfied.

In the third section we show that under suitable restrictions on $T$, the properties of $\tilde{X}_{T}$ are enjoyed locally. This does not follow from the general arguments used in the second section, because there the construction of an annihilating measure for $R\left(\tilde{X}_{T}\right)$ is not local.

Let $T \subseteq\{|z|<1\}$ be a "Swiss cheese" in a sense to be made precise later. (The previously mentioned example of Browder satisfies our definition.)

Theorem 4. If $K$ is a compact subset of $\tilde{X}_{T}$ with nonempty interior in the topology of $\widetilde{X}_{T}$, then the restriction of $R\left(\widetilde{X}_{T}\right)$ to $K$ is not dense in $C(K)$.

In the fourth and concluding section we demonstrate that no smoothness assumptions alone can be sufficient to avoid the pathological behavior exhibited by the sets $X_{S}$ and $\widetilde{X}_{T}$ discussed in the first two sections. Here we will be interested in the algebra $P(X)$ and its maximal ideal space $b(X)$, defined for a compact set $X \subseteq \mathbf{C}^{n}$ as follows:

$P(X)$ is the uniform closure in $C(X)$ of the polynomials in the coordinate functions $z_{1}, \cdots, z_{n}$.

$b(X)$, the polynomially convex hull of $X$, is the set $\left\{z \in \mathbf{C}^{n} \mid\right.$ for all polynomials $p$ in $\left.z_{1}, \cdots, z_{n},|p(z)| \leq \max _{X}|p|\right\}$.

Starting with the example in [7], or with the alternative use of Wermer's construction on p. 365 , we prove the following.

Theorem 5. There is a $C^{\infty} 3$-sphere $\Sigma_{1}$ contained in $\mathbf{C}^{6}$ such that $b\left(\Sigma_{1}\right)$ contains points not in $\Sigma_{1}$ but does not contain analytic structure.

We obtain a similar result by starting instead with the set $\widetilde{X}_{T}$ of the second section.

Theorem 6. There is a $C^{\infty}$ 3-sphere $\Sigma_{2}$ contained in $\mathbf{C}^{6}$ such that $h\left(\Sigma_{2}\right)=\Sigma_{2}$, every point of $\Sigma_{2}$ is a peak point for $P\left(\Sigma_{2}\right)$, but $P\left(\Sigma_{2}\right) \neq C\left(\Sigma_{2}\right)$. In other words $P\left(\Sigma_{2}\right)$ satisfies the above conditions (I), (II) and (III).

1. We will employ the following notation throughout:

$\Delta$ will denote the closed unit disk; $\Delta^{2}$ is then the closed unit bicylinder $\left\{(z, w) \in \mathbf{C}^{2}|| z|\leq 1| w \mid, \leq 1\right\}$

$\partial X$ will denote the topological boundary of a set $X \subseteq \mathrm{C}, \mathrm{C}^{2}$ or $\mathrm{C}^{n}$ depending on the context;

$X_{T}$, where $T \subseteq \Delta$, will denote the set $\left\{(z, w) \in \Delta^{2} \mid z \in T\right.$ and $|w|=1$ or $|z|=$ 1 and $w \in T\}$ 
$S$ (or $S_{n}$ ) will denote a closed connected subset of $\Delta$ such that $\partial \Delta \subseteq S$ (or $S_{n}$ ).

The following lemma, which is stated in a slightly different form in [7], will be useful to us:

Lemma 1. Fix $p \in \Delta^{2}$. If $p \notin b_{r}\left(X_{S}\right)$, then $p \in b(K)$ for some compact set $K \subseteq X \Delta \backslash s^{*}$

Proof. If $p \notin h_{r}\left(X_{S}\right)$, then there is some polynomial $P$ such that $P(p)=0$, but $P$ has no zero on $X_{S}$. Let $V$ be the component of $\left\{x \in \mathbf{C}^{2} \mid P(x)=0\right\}$ which contains $p$, and let $K=V \cap \partial \Delta^{2}$. By assumption, $K \cap X_{S}=\varnothing$, so that $K \subseteq\left\{(z, w) \in \partial \Delta^{2} \mid z \notin S\right.$ or $\left.w \notin S\right\}=X \Delta \backslash S^{\circ}$. By the maximum principle, if $Q$ is any polynomial on $\mathbf{C}^{2}$, then $|Q(p)| \leq \sup _{x \in K}|Q(x)|$, so $p \in b(K)$ as claimed.

Definition. Given $S$ as above, it is possible to choose a sequence $\left\{S_{n}\right\}_{n=1}^{\infty}$ of closed connected subsets of $\Delta$ which contain $\partial \Delta$ and satisfy

(i) $S_{n}$ is bounded by finitely many disjoint smooth curves;

(ii) $S_{n}$ is a neighborhood of $S_{n+1}$ in $\Delta$;

(iii) $S=\bigcap_{n=1}^{\infty} S_{n}$.

Let $u_{n}$ be the unique function in $C\left(S_{n}\right)$ which is harmonic on int $S_{n}$ and satisfies $u_{n}\left|\partial \Delta \equiv 0, u_{n}\right| \partial S_{n} \backslash \partial \Delta \equiv 1$. For $z \in S$ we define $u_{S}(z)=\lim _{n \rightarrow \infty} u_{n}(z)$. By the maximum principle the functions $u_{n}$ are monotone increasing, so $u_{s}(z)$ exists for each $z \in S$. For future reference note that $u_{S}(z)>0$ on $S \backslash \partial \Delta$ whenever $S \neq \Delta$.

Lemma 2. $u_{S}$ is independent of the choice of $\left\{S_{n}\right\}$.

Proof. Let $\left\{S_{m}^{\prime}\right\}_{m=1}^{\infty}$ be another sequence of compact sets as above, and let $\left\{u_{m}^{\prime}\right\}$ be the corresponding functions in $C\left(S_{m}^{\prime}\right)$.

Given any $n, S_{n}$ is a neighborhood in $\Delta$ of $S_{n+1}$, hence also of $S$. Since $\bigcap_{m=1}^{\infty} S_{m}^{\prime}=S$, there is an integer $m_{0}$ such that $S_{n}$ is a neighborhood of $S_{m_{0}}^{\prime}$; in particular, $\partial S_{m_{0}}^{\prime} \subseteq S_{n}$, so $u_{m_{0}}^{\prime} \geq u_{n}$ on $S_{m_{0}}^{\prime}$. Thus we have for $z \in S$ :

$$
\lim _{m \rightarrow \infty} u_{m}^{\prime}(z) \geq u_{m}^{\prime}(z) \geq u_{n}(z)
$$

Since $n$ was arbitrary, $\lim _{m \rightarrow \infty} u_{m}^{\prime}(z) \geq \lim _{n \rightarrow \infty} u_{n}(z)=u_{S}(z)$. Similarly, $\lim _{m \rightarrow \infty} u_{m}^{\prime}(z) \leq u_{S}(z)$ for all $z \in S$.

Observe that if $S$ is "nice," then $u_{S}$ has the following properties.

Lemma 3. If $S$ is bounded by a finite number of disjoint smooth curves, then $u_{S} \in C(S), u_{s}$ is barmonic on int $S$, and $u_{S}\left|\partial \Delta \equiv 0, u_{S}\right| \partial S \backslash \partial \Delta \equiv 1$.

Proof. Pick $\left\{S_{n}\right\}$ satisfying (i), (ii), (iii) in the definition of $u_{s}$, and let $\left\{u_{n}\right\}$ be the corresponding functions used to define $u_{S}$. Let $u \in C(S)$ be harmonic on 
int $S$ and satisfy $u|\partial \Delta \equiv 0, u| \partial S \backslash \partial \Delta \equiv 1$. Since $S$ is smoothly bounded, there is a sequence of harmonic functions $\left\{v_{m}\right\}$ each defined in some neighborhood of $S$, such that $v_{m}$ converges to $u$ uniformly on $S$ (see for example, [9, p. 184]).

If $z \in S$, then for all $n, u_{n}(z) \leq u(z)$ by the maximum principle, so $u_{S}(z) \leq$ $u(z)$. To prove the converse, we may assume that $v_{m} \leq 0$ on $\partial \Delta$ and $v_{m} \leq 1-1 / m$ on $S$. Given any $m$, we choose $n$ sufficiently large that $S_{n} \subseteq\left\{v_{m} \leq 1\right\}$; then $v_{m}\left|\partial S_{n} \leq u_{n}\right| \partial S_{n}$, so that $v_{m}(z) \leq u_{n}(z) \leq u_{S}(z)$. Since $m$ was arbitrary, $u(z)=$ $\lim _{m \rightarrow \infty} v_{m}(z) \leq u_{S}(z)$, whence $u_{S}=u$.

Our main result may now be stated.

Theorem 1. $h_{r}\left(X_{S}\right)=\left\{(z, w) \in S \times S \mid u_{S}(z)+u_{S}(w) \leq 1\right\}$.

We first prove two lemmas.

Lemma 4. Let $B$ be the annulus $\{z \in \mathrm{C}|1 \geq| z \mid \geq r\}$. Then $b_{r}\left(X_{B}\right)=$ $\left\{(z, w) \in \Delta^{2}|| z w \mid \geq r\right\}$. (This is a special case of Theorem 1, since by Lemma 3, $u_{B}=(\log |z|) /(\log r)$. It is also a special case of a similar theorem for "circled" subsets of $\mathrm{C}^{n}$, referred to in the introduction.)

Proof. Fix $\left(z_{0}, w_{0}\right) \in \Delta^{2}$. If $\left|z_{0} w_{0}\right|<r$, the polynomial $P(z, w)=z w-z_{0} w_{0}$ vanishes at $\left(z_{0}, w_{0}\right)$, but has no zero on $X_{B}$, so $\left(z_{0}, w_{0}\right) \notin b_{r}\left(X_{B}\right)$.

Conversely, suppose that $\left|z_{0} w_{0}\right| \geq r$. The polynomial $P(z, w)=z w$ is larger in modulus at $\left(z_{0}, w_{0}\right)$ than it is on any compact subset of $X_{\Delta \backslash B^{*}}$. By Lemma 1 , $\left(z_{0}, w_{0}\right) \in b_{r}\left(X_{B}\right)$.

Lemma 5. Let $X$ be a compact subset of $\mathrm{C}^{n}$ and let $\Phi: X \rightarrow \mathrm{C}^{m}$. Suppose that $z_{i} \circ \Phi \in R(X)$ for each coordinate projection $z_{i}, i=1,2, \cdots, m$. Then $\Phi\left(b_{r}(X)\right) \subseteq b_{r}(\Phi(X))$.

Proof. Fix $p_{0} \in b_{r}(X)$, and suppose that $P$ is a polynomial on $\mathbf{C}^{m}$ such that $P\left(\Phi\left(p_{0}\right)\right)=0$. We must show that $P$ has a zero on $\Phi(X)$.

Any function $F \in R(X)$ which has a zero on $b_{r}(X)$ must have a zero on $X$. Indeed, suppose $F(p)=0$ for some $p \in b_{r}(X)$. Choose $f_{n} \in R_{0}(X)$ such that $\sup _{X}\left|F-f_{n}\right| \leq 1 / n$. Let $g_{n}=f_{n}-f_{n}(p)$, then $g_{n}(p)=0$, and $g_{n}=P_{n} / Q_{n}$ for some polynomials $P_{n}, Q_{n}$ with $Q_{n} \neq 0$ on $X$ hence on $b_{r}(X)$. So $P_{n}(p)=0$, and since $p \in b_{r}(X), P_{n}\left(p_{n}\right)=0$ for some $p_{n} \in X$. Then $g_{n}\left(p_{n}\right)=0$, so that

$$
\begin{aligned}
\left|F\left(p_{n}\right)\right| & \leq\left|F\left(p_{n}\right)-f_{n}\left(p_{n}\right)\right|+\left|f_{n}\left(p_{n}\right)-f_{n}(p)\right|+\left|f_{n}(p)-F(p)\right| \\
& \leq 1 / n+\left|g_{n}\left(p_{n}\right)\right|+1 / n=2 / n .
\end{aligned}
$$

Some subsequence $p_{n_{k}} \rightarrow p_{\infty} \in X$, so that $F\left(p_{\infty}\right)=0$ for some $p_{\infty} \in X$ as claimed. 
In our case, $P(\Phi) \in R(X)$ and $P(\Phi)\left(p_{0}\right)=0$, so we conclude that $P(\Phi)(p)=0$ for some $p \in X$. Thus $P$ has a zero at $\Phi(p) \in \Phi(X)$ as desired.

Corollary. Let $S$ be finitely connected. Suppose that $\phi$ is a one to one continuous map from $S$ into $\Delta$ such that $\phi(\partial \Delta)=\partial \Delta$ and $\phi$ is bolomorpbic on int $S$. Define $\Phi: h_{r}\left(X_{S}\right) \rightarrow \Delta^{2}$ by $\Phi(z, w)=(\phi(z), \phi(w))$. Then $\Phi\left(b_{r}\left(X_{S}\right)\right)=b_{r}\left(X_{\phi(S)}\right)$.

Proof. $\phi \in R(S)$ since $S$ is finitely connected (see, for example, [9, p. 231] or [3, p. 51]), and similarly $\phi^{-1} \in R(\phi(S))$. Since evidently $X_{S} \subseteq S \times S$, $b_{r}\left(X_{S}\right) \subseteq S \times S$, so that $z \circ \Phi$ and $w \circ \Phi$ are in $R\left(b_{r}\left(X_{S}\right)\right)$; similarly $z \circ \Phi^{-1}$ and $w \circ \Phi^{-1}$ are in $R\left(b_{r}\left(X_{\phi(S)}\right)\right)$. Observe that

$$
\begin{aligned}
X_{\phi(S)} & =\{(\zeta, \eta)|| \zeta \mid=1, \eta \in \phi(S) \quad \text { or }|\eta|=1, \zeta \in \phi(S)\} \\
& =\{(\phi(z), \phi(w))|| z \mid=1, w \in S \quad \text { or }|w|=1, z \in S\}=\Phi\left(X_{S}\right) .
\end{aligned}
$$

Lemma 5 applied to $\Phi$ yields

$$
\Phi\left(b_{r}\left(X_{S}\right)\right) \subseteq b_{r}\left(\Phi\left(X_{S}\right)\right)=h_{r}\left(X_{\phi(S)}\right)
$$

and applying Lemma 5 to $\Phi^{-1}$ we have conversely

$$
\begin{aligned}
b_{r}\left(X_{\phi(S)}\right) & =\Phi\left(\Phi^{-1}\left[b_{r}\left(X_{\phi(S)}\right)\right]\right. \\
& =\Phi\left(\Phi^{-1}\left[b_{r}\left(\Phi\left(X_{S}\right)\right)\right]\right) \subseteq \Phi\left(b_{r}\left(\Phi^{-1} \circ \Phi\left(X_{S}\right)\right)\right)=\Phi\left(b_{r}\left(X_{S}\right)\right) .
\end{aligned}
$$

Proof of Theorem 1. We first prove the theorem for those $S$ of the form $S=$ $\Delta \backslash \bigcup_{i=1}^{n}$ int $D_{i}$, where the $D_{i}$ are disjoint closed disks in int $\Delta ;$ then we shall deduce the general case by a "limit" argument. To avoid triviality we assume $n>0$. In the simplest case $n=1$, we can reduce the problem to the situation of Lemma 4 by mapping $S$ onto an annulus via a linear fractional transformation, and applying the corollary to Lemma 5. For $n>1$ we would like to imitate this procedure by defining $\phi: S \rightarrow\{1 \geq|\zeta| \geq r\}$ such that $\phi(\partial \Delta)=\{|\zeta|=1\}$ and $\phi\left(\partial D_{i}\right)=$ $\{|\zeta|=r\}$ for $i=1,2, \cdots, n$, with $\phi$ analytic on int $S$. Although in general one can only construct a $\phi$ with approximately these properties-and, of course, $\phi$ will not be $1-$ to-1-it turns out that we can again reduce the problem to the situation of Lemma 4 .

Fix $(a, b) \in S \times S$.

Case 1. Suppose that $u_{S}(a)+u_{S}(b)<1$. We will show that $(a, b) \in_{r}\left(X_{S}\right)$ by proving that any polynomial which vanishes at $(a, b)$ also has a zero on $X_{S}$. So let $P(a, b)=0$, and let 


$$
\delta=1-u_{S}(a)-u_{S}(b)>0 .
$$

For convenience we divide the proof of Case 1 into three steps.

Step A: Construction of $\phi$. We shall construct a $\phi_{\delta}$-call it $\phi$-which approximate ly maps $S$ onto an annulus.

Let $u_{i}$ be the continuous function on $S$ which is harmonic on int $S$ and satisfies:

$$
u_{i}\left|\partial D_{i} \equiv 1, \quad u_{i}\right| \partial S \backslash \partial D_{i} \equiv 0 ; \quad i=1,2, \cdots, n
$$

Note that $u_{S}=\Sigma_{i=1}^{n} u_{i}$ by Lemma 3. Each $u_{i}$ extends to be harmonic in a neighborhood of $S$ by the reflection principle. In this way we obtain an open set $\mathcal{O} \supseteq S$ which we may take small enough that

(i) $\sum_{i=1}^{n}\left|u_{i}(z)\right|<2$ for $z \in \mathcal{O}$;

(ii) $u_{s}(z)>1$ for $z \in \mathcal{O} \cap$ int $D_{i}, i=1,2, \cdots, n$;

(iii) $u_{i}(z)<0$ for $z \in \mathcal{O} \backslash \Delta, i=1,2, \cdots, n$.

We may also assume that $\partial \mathcal{O}$ consists of circles, one in each component of $C \backslash S$.

Let $\gamma_{0}:[0,1] \rightarrow \mathcal{O}$ be a positively oriented circle contained in $\Theta \backslash \Delta$.

Similarly, let $\gamma_{j}$ be a positively oriented circle contained in $\Theta \cap$ int $D_{j}, j=1,2$, $\cdots, n$. To avoid confusion we will distinguish between the map $\gamma_{j}$ and its image which we will denote by $\Gamma_{j}, j=0,1, \cdots, n$. If $\gamma$ is any closed curve in $\Theta$, then $\gamma$ is homologous in $\Theta_{\text {to }} \Sigma_{j=1}^{n} c_{j} \gamma_{j}$ for some integers $c_{1}, \cdots, c_{n}$.

Let $v_{S}$ be the (multiple-valued) conjugate harmonic function to $u_{S}$, and let $v_{i}$ be the harmonic conjugate of $u_{i}, i=1,2, \cdots, n$. Define

$$
a_{j}=\frac{1}{2 \pi} \underset{\gamma_{j}}{\operatorname{var}} v_{S}, \quad \omega_{i j}=\frac{1}{2 \pi} \operatorname{var}_{j} v_{i} .
$$

Observe that $\operatorname{det}\left(\left(\omega_{i j}\right)\right) \neq 0$. If not, there would be real constants $c_{i}$, not all zero, such that

$$
\sum_{i=1}^{n} c_{i} \omega_{i j}=0 \text { for } j=1,2, \cdots, n \text {. }
$$

Then $f=\sum_{i=1}^{n} c_{i}\left(u_{i}+i v_{i}\right)$ would be a nonconstant single-valued analytic function on $\mathcal{O}$, with

$$
f(\partial S) \subseteq\{\operatorname{Re} z=0\} \cup\left\{\operatorname{Re} z=c_{1}\right\} \cup \ldots \cup\left\{\operatorname{Re} z=c_{n}\right\} .
$$

As this is absurd, we conclude that there are indeed constants $\eta_{k i}, 1 \leq k, i \leq n$, such that $\Sigma_{i=1}^{n} \eta_{k i} \omega_{i j}=\delta_{k}^{j}, 1 \leq j, k \leq n$.

Now choose real numbers $\beta_{1}, \cdots, \beta_{n}$ in such a way that $\alpha_{j}+\beta_{j}$ is rational 
for each $j$, making sure that the $\beta_{j}$ are sufficiently small that if we let $d_{i}=$ $\sum_{k=1}^{n} \beta_{k} \eta_{k i}$, then

$$
K=\underset{i}{2 \max _{i}}\left|d_{i}\right|<\delta / 2
$$

Now we define $u$ on $\mathcal{O}$ by

$$
u=u_{S}+\sum_{i=1}^{n} d_{i} u_{i}=\sum_{i=1}^{n}\left(1+d_{i}\right) u_{i}
$$

The harmonic conjugate of $u$ is $v=v_{S}+\sum_{i=1}^{n} d_{i} v_{i}$, so

$$
\begin{aligned}
\frac{1}{2 \pi} \gamma_{j} \operatorname{var} v & =\frac{1}{2 \pi} \operatorname{var}_{j} v_{S}+\frac{1}{2 \pi} \sum_{i=1}^{n} d_{i} \underset{\gamma_{j}}{\operatorname{var}} v_{i}=a_{j}+\sum_{i=1}^{n} d_{i} \omega_{i j} \\
& =\alpha_{j}+\sum_{i=1}^{n} \sum_{k=1}^{n} \beta_{k} \eta_{k i} \omega_{i j}=\alpha_{j}+\sum_{k=1}^{n} \beta_{k} \delta_{k}^{j}=\alpha_{j}+\beta_{j} .
\end{aligned}
$$

Choose a positive integer $N$ large enough that $N\left(\alpha_{j}+\beta_{j}\right)$ is an integer for $j=1,2, \cdots, n$, and let $\phi=e^{-N(u+i v)}$. By construction, $\phi$ is a single-valued analytic function on $\Theta$.

Step B: Properties of $\phi$. Let $B=\left\{\zeta \in \mathrm{C}|1 \geq| \zeta \mid \geq e^{-N(1-K)}\right\}$. We claim that $\phi^{-1}(B) \subseteq S$. Note that for $z \in \phi^{-1}(B)$ we have $0 \leq u(z) \leq 1-K$, and suppose that $z \in \mathcal{O} \backslash S$. There are two cases to consider.

If $z \in \mathcal{O} \backslash \Delta$, then $u_{i}(z)<0$ for $i=1,2, \cdots, n$ by condition (iii) above. Note that $\delta \leq 1$ by equation (1), so that

$$
u(z)=\sum_{i=1}^{n}\left(1+d_{i}\right) u_{i}(z)<\sum_{i=1}^{n}(1-\delta / 4) u_{i}(z)<0
$$

by equations (2) and (3). Thus $z \notin \phi^{-1}(B)$.

If $z \in$ int $D_{j} \cap \mathcal{O}$, then

$$
\begin{aligned}
u(z) & =u_{S}(z)+\sum_{i=1}^{n} d_{i} u_{i}(z) \\
& \geq u_{S}(z)-\sum_{i=1}^{n}\left|d_{i}\right|\left|u_{i}(z)\right|>u_{S}(z)-K / 2 \cdot 2 \geq 1-K
\end{aligned}
$$

this follows from equations (2) and ( 3 ) and conditions (i) and (ii) above. Again, $z \notin \phi^{-1}(B)$. 
In particular, we have established that $\phi^{-1}(\{|\zeta|=1\})$ is contained in $S$. But if $z \in S \backslash \partial \Delta$, then $u_{i}(z) \geq 0$ for $i=1,2, \cdots, n$, and $u_{S}(z)>0$ as noted earlier, so that

$$
u(z)=\sum_{i=1}^{n}\left(1+d_{i}\right) u_{i}(z) \geq(1-\delta / 4) u_{S}(z)>0
$$

Thus $\phi^{-1}(\partial \Delta) \subseteq \partial \Delta$.

Now let $U$ be the subset of $\mathcal{O}$ bounded by the images $\Gamma_{j}$ of the maps $\gamma_{j}$, $j=0,1, \cdots, n$. Let

$$
R=\inf _{\boldsymbol{z} \in \Gamma_{0}} e^{-N u(z)}, \quad r=\sup _{z \in \Gamma_{j} ; 1 \leq j \leq n} e^{-N u(z)} .
$$

$\Gamma_{0} \subseteq \mathcal{O} \backslash \Delta$, so by the above $R>1$. Similarly, $\Gamma_{j} \subseteq \mathcal{O} \cap$ int $D_{j}$, so that $r<e^{-N(1-K)}<1$. Let

$$
U^{\prime}=\left\{z \in U \mid R>e^{-N u(z)}>r\right\}
$$

and note that $\phi\left(U^{\prime}\right) \supseteq B$, since $\phi\left(\partial U^{\prime}\right)=\{|\zeta|=r\} \cup\{|\zeta|=R\}$.

For $\zeta \in \phi(\Theta)$ let $m(\zeta)$ denote the number of times including multiplicities that $\phi$ takes on the value $\zeta$ in $U . m(\zeta)$ is constant on each component of $\phi(\mathcal{O}) \backslash \bigcup_{j=0}^{n} \phi\left(\Gamma_{j}\right)$. By choice of $r$ and $R$, the set $\{\zeta \in \mathrm{C}|r<| \zeta \mid<R\}$ is contained in a component of $\phi(\theta) \backslash \bigcup_{j=0}^{n} \phi\left(\Gamma_{j}\right)$. Thus $m(\zeta)$ is constant on $\phi\left(U^{\prime}\right)$; we use $m$ to denote this constant value. $m>0$ since if $z_{0} \in \partial \Delta$, then $r<1=\left|\phi\left(z_{0}\right)\right|<R$.

We conclude that for all $\zeta \in \phi\left(U^{\prime}\right), \zeta$ has precisely $m$ inverses in $U^{\prime}$ under $\phi$. Call these inverses $\psi_{1}(\zeta), \cdots, \psi_{m}(\zeta)$ in any order.

Step C: Reduction to Lemma 4. Recall that we are trying to show that a given polynomial $P$ which vanishes at $(a, b)$ also has a zero on $X_{S}$. We are now ready to use $P$ to define a function $Q \in R\left(X_{B}\right)$. In fact, let

$$
Q(\zeta, \eta)=\prod_{i, j=1}^{m} P\left(\psi_{i}(\zeta), \psi_{j}(\eta)\right), \quad(\zeta, \eta) \in \phi\left(U^{\prime}\right) \times \phi\left(U^{\prime}\right)
$$

Note that $Q$ is well defined since it is independent of the numbering of the $\psi_{i}$. Fix $\eta$. Except for $\zeta$ in a discrete subset of $\phi\left(U^{\prime}\right), \zeta$ will have $m$ distinct inverse images in $U^{\prime}$ under $\phi$. For such $\zeta$ one may renumber the $\psi_{i}$ near $\zeta$ to make them analytic near $\zeta$. Thus $Q(\zeta, \eta)$ is analytic in $\zeta$ except possibly on a discrete set. For any of the isolated points $\zeta_{0}$ with less than $m$ distinct inverse images, $\zeta \rightarrow$ $\Pi_{i, j=1}^{m} P\left(\psi_{i}(\zeta), \psi_{j}(\eta)\right)$ is analytic and bounded in a deleted neighborhood of $\zeta_{0}$, 
and so extends to be analytic at $\zeta_{0}$. Similarly, for fixed $\zeta$ the function $\eta \rightarrow$ $\Pi_{i, j=1}^{m} P\left(\psi_{i}(\zeta), \psi_{j}(\eta)\right)$ is analytic on $\phi\left(U^{\prime}\right)$, so that $Q$ is analytic on $\phi\left(U^{\prime}\right) \times$ $\phi\left(U^{\prime}\right)$. Now it was noted previously that $B \subseteq \phi\left(U^{\prime}\right)$, so $X_{B} \subseteq B \times B \subseteq \phi\left(U^{\prime}\right) \times$ $\phi\left(U^{\prime}\right)$. Since $B \times B$ is rationally convex, $Q \in R\left(X_{B}\right)$.

Observe now that

$$
\begin{array}{rlrl}
|\phi(a) \phi(b)| & =|\exp \{-N(u(a)+i v(a))-N(u(b)+i v(b))\}| & & \\
& =\exp \{-N(u(a)+u(b))\} & \\
& \geq \exp \left\{-N\left[\sum_{i=1}^{n}\left(1+\left|d_{i}\right|\right)\left(u_{i}(a)+u_{i}(b)\right)\right]\right\} & & \text { by }(3) \\
& \geq \exp \left\{-N\left[(1+K / 2)\left(u_{S}(a)+u_{S}(b)\right)\right]\right\} & & \text { by (2) } \\
& \geq \exp \{-N[1-\delta+K / 2]\} & & \text { by (1) } \\
& \geq \exp \{-N[1-K]\} & & \text { by (2). }
\end{array}
$$

By Lemma $4, b_{r}\left(X_{B}\right)=\left\{(\zeta, \eta) \in \Delta^{2}|1 \geq| \zeta \eta \mid \geq e^{-N(1-K)}\right\}$, so we have that $(\phi(a), \phi(b)) \in b_{r}\left(X_{B}\right)$. By definition of the $\psi_{i}$ there exist $i_{0}$ and $j_{0}$ such that $\psi_{i_{0}}(\phi(a))=a$ and $\psi_{j_{0}}(\phi(b))=b$. (Note that $\psi_{i}(\phi(a))$ and $\psi_{j}(\phi(b))$ are well defined for all $i$ and $j$, since $(\phi(a), \phi(b)) \in b_{r}\left(X_{B}\right)$ implies $\phi(a), \phi(b) \in B$, and so by the fact that $B \subseteq \phi\left(U^{\prime}\right)$ we have $\phi(a), \phi(b) \in \phi\left(U^{\prime}\right)$ as desired.) Thus,

$$
Q(\phi(a), \phi(b))=P(a, b) \prod_{i \neq i_{0} \text { or } j \neq j_{0}} P\left(\psi_{i}(\phi(a)), \psi_{j}(\phi(b))\right)=0 .
$$

It follows that there is some point $\left(\zeta_{0}, \eta_{0}\right) \in X_{B}$ such that $Q\left(\zeta_{0}, \eta_{0}\right)=0$, as was show $n$ in the proof of Lemma 5. Hence

$$
\prod_{i, j=1}^{m} P\left(\psi_{i}\left(\zeta_{0}\right), \psi_{j}\left(\eta_{0}\right)\right)=0
$$

so that for some $i_{1}, j_{1}$ we have $P\left(\psi_{i_{1}}\left(\zeta_{0}\right), \psi_{j_{1}}\left(\eta_{0}\right)\right)=0$. As noted earlier, $\phi^{-1}(\partial \Delta) \subseteq \partial \Delta$ and $\phi^{-1}(B) \subseteq S$, so $\left(z_{0}, w_{0}\right)=\left(\psi_{i_{1}}\left(\zeta_{0}\right), \psi_{j_{1}}\left(\eta_{0}\right)\right) \in X_{S}$. Thus $P$ has the zero $\left(z_{0}, w_{0}\right) \in X_{S}$ as claimed.

Case 2. Suppose that $u_{s}(a)+u_{s}(b)>1$. Let

$$
\delta=u_{s}(a)+u_{s}(b)-1>0 .
$$

Proceed to define $\phi$ exactly as in Case 1. Now, however, let

$$
B^{\prime}=\left\{\zeta \in \mathbf{C}|1 \geq| \zeta \mid \geq e^{-N(1+K)}\right\},
$$


a larger annulus than before. In fact, if $z \in S$ then $\phi(z) \in B^{\prime}$ because

$$
\begin{aligned}
|\phi(z)| & =\exp \{-N u(z)\}=\exp \left\{-N\left(u_{S}(z)+\sum_{i=1}^{n} d_{i} u_{i}(z)\right)\right\} \\
& \geq \exp \left\{-N\left(u_{S}(z)+K / 2\right)\right\} \geq \exp \{-N(1+K / 2)\}
\end{aligned}
$$

by using equations (2) and (3) which are still valid.

Now define $\Phi(z, w)=(\phi(z), \phi(w))$ for $(z, w) \in \mathcal{O}_{\times} \mathcal{O}$. $\Phi$ is analytic on $\mathcal{O}_{\times} \mathcal{O}$, so $z \circ \Phi$ and $w \circ \Phi$ are in $R\left(X_{S}\right)$. We have

$$
\begin{array}{rlrl}
|\phi(a) \phi(b)| & =\exp \{-N(u(a)+u(b))\} & \\
& \leq \exp \left\{-N\left(u_{S}(a)+u_{S}(b)-\sum_{i=1}^{n}\left|d_{i}\right|\left(u_{i}(a)+u_{i}(b)\right)\right)\right\} & & \text { by }(3) \\
& \leq \exp \{-N(1+\delta-K)\} & & \text { by }\left(1^{\prime}\right),(2) \\
& <\exp \{-N(1+K)\} & \text { by }(2)
\end{array}
$$

Thus by Lemma $4, \Phi(a, b) \notin b_{r}\left(X_{B^{\prime}}\right)$. The remark at the end of the preceding paragraph shows that $\phi(S) \subseteq B^{\prime}$; as in Case $1, \phi(\partial \Delta)=\partial \Delta$, so $\Phi\left(X_{s}\right)=X_{\phi(s)} \subseteq X_{B^{\prime}}$. Thus $\Phi(a, b) \notin b_{r}\left(\Phi\left(X_{s}\right)\right)$. By Lemma $5,(a, b) \notin b_{r}\left(X_{S}\right)$.

Case 3. Suppose, finally, that $u_{S}(a)+u_{s}(b)=1$. We may assume that $u_{S}(a)>0$. Choose $a_{n} \in S$ with $u_{S}\left(a_{n}\right)<u_{s}(a)$ and $a_{n} \rightarrow a$. We have $\left(a_{n}, b\right) \rightarrow$ $(a, b)$, and each $\left(a_{n}, b\right) \in b_{r}\left(X_{s}\right)$, since $u_{s}\left(a_{n}\right)+u_{s}(b)<1$. Since $b_{r}\left(X_{s}\right)$ is closed, $(a, b) \in b_{r}\left(X_{s}\right)$.

Given an arbitrary set $S$, one can find a sequence of regions $S_{n}$ with properties (i), (ii) and (iii) in the definition of $u_{s}$ following Lemma 1. For each $n$, int $S_{n}$ is conformally equivalent to a domain bounded by circles; the equivalence extends to a continuous 1 to 1 map on $S_{n}$. By the corollary to Lemma 5 and the special case above, the theorem holds for each $S_{n}$.

Since $S \subseteq S_{n}$ for each $n, b_{r}\left(X_{S}\right) \subseteq \bigcap_{n=1}^{\infty} b_{r}\left(X_{S_{n}}\right)$. Conversely, suppose that $p \in b_{r}\left(X_{s_{n}}\right)$ for all $n$. If $P$ is any polynomial which vanishes at $p$, then there is some point $p_{n} \in X_{S}$ such that $P\left(p_{n}\right)=0$. By compactness there is a subsequence $p_{n_{k}}$ converging to $p_{0}^{n} \in \bigcap_{n=1}^{\infty} X_{S}=X_{S}$, and clearly $P\left(p_{0}\right)=0$. Thus $p \in b_{r}\left(X_{S}\right)$. So we have $b_{r}\left(X_{s}\right)=\bigcap_{n=1}^{\infty} b_{r}\left(X_{s_{n}^{n}}^{n}\right)$. Now $u_{s}(z)=\lim _{n \rightarrow \infty} u_{s_{n}}(z)$ for $z \in S$, and the $u_{S_{n}}$ are monotone increasing, so 


$$
\begin{aligned}
b_{r}\left(X_{S}\right) & =\bigcap_{n=1}^{\infty} b_{r}\left(X_{S_{n}}\right)=\bigcap_{n=1}^{\infty}\left\{(z, w) \in S_{n} \times S_{n} \mid u_{S_{n}}(z)+u_{S_{n}}(w) \leq 1\right\} \\
& =\left\{(z, w) \in S \times S \mid u_{S}(z)+u_{S}(w) \leq 1\right\}
\end{aligned}
$$

and the theorem is proven.

Note 1 . The result can, of course, be generalized somewhat. For example, by modifying Lemma 4 and the proof of Theorem 1, one can show

$$
\begin{aligned}
& \text { given } S_{1}, S_{2}, \cdots, S_{n} \text { then } b_{r}\left(S_{1} \times S_{2} \times \cdots \times S_{n} \cap \partial \Delta^{n}\right) \\
& =\left\{z \in S_{1} \times S_{2} \times \cdots \times S_{n} \mid u_{S_{1}}\left(z_{1}\right)+\cdots+u_{S_{n}}\left(z_{n}\right) \leq n-1\right\}
\end{aligned}
$$

Note 2. The restriction to sets $S$ which are connected is not serious, since more general subsets of $\Delta$ are of no additional interest. In fact, if $\partial \Delta \subseteq T \subseteq \Delta$ and $T$ is closed, let $S$ be the component of $\partial \Delta$ in $T$. One readily verifies that

$$
b_{r}\left(X_{T}\right)=b_{r}\left(X_{S}\right) \cup X_{T} \text {, }
$$

so that the other components of $T$ contribute nothing to $b_{r}\left(X_{T}\right) \backslash X_{T}$. We outline a proof.

Choose nonempty compact sets $K_{n} \subseteq \Delta \backslash S$ such that $K_{n+1} \supseteq K_{n}$ and $\bigcup_{n=1}^{\infty} K_{n}=\Delta \backslash S$. One can then choose smoothly bounded compact sets $C_{n}, D_{n}$ such that $T \subseteq C_{n} \cup D_{n}, S \subseteq C_{n}, K_{n} \subseteq D_{n}$, and $C_{n} \cap D_{n}=\varnothing$. Let $l_{n}$ be a finite union of line segments which meet $C_{n}$ and $D_{n}$ precisely at the ir endpoints, chosen so that the compact set $S_{n}$ defined by $S_{n}=C_{n} \cup D_{n} \cup l_{n}$ is connected.

Observe that $u_{S} \equiv 1$ on $D_{n} \cup l_{n}$. As remarked earlier, $u_{S}{ }_{n}>0$ on $S_{n} \backslash \partial \Delta$, so, by Theorem $1, b_{r}\left(X_{S_{n}}\right)=b_{r}\left(X_{C_{n}}\right) \cup X_{S_{n}}$. The result (4) follows by using the facts that $T \subseteq S_{n}$ and that $\bigcap_{n=1}^{\infty} C_{n}^{n}=S$.

Example. In [7], Wermer shows that $h_{r}\left(X_{S}\right)$ does not "contain analytic structure" precisely when int $S=\varnothing$. He proceeds to construct a specific $S$ such that $b_{r}\left(X_{S}\right) \neq X_{S}$, although int $S=\varnothing$. Using Theorem 1 we have an alternative method for constructing such an $S$.

If $|a|<1$ and $0<r<1-|a|$, let $D(a, r)=\{|z-a|<r\}$. Then $u_{\Delta \backslash D(a, r)}(z) \rightarrow$ 0 as $r \rightarrow 0$ if $z \in \Delta \backslash\{a\}$. (For example, if $a=0$, then $u \Delta \backslash D(a, r)(z)=\log |z| / \log r$ and this assertion is clear; the general case can be essentially reduced to the case $a=0$ by appropriate linear fractional transformations.)

Let $\left\{a_{j}\right\}$ be a countable dense subset of int $\Delta$, and let $b$ be any other point of int $\Delta$. Take $D_{1}$ to be an open disk centered at $a_{1}$, such that $\bar{D}_{1} \subseteq$ int $\Delta$ and $u_{\Delta \backslash D_{1}}(b) \leq 1 / 4$; this is possible by the preceding remark. Suppose that 
$D_{1}, D_{2}, \cdots, D_{n-1}$ have been chosen. Let $a$ be the first of the $a_{j}$ which is not in $\bigcup_{i=1}^{n-1} \bar{D}_{i}$. Choose an open disk $D_{n}$ with center a such that $\bar{D}_{n} \subseteq$ int $\Delta \backslash \bigcup_{i=1}^{n-1} \bar{D}_{i}$ and $u_{\Delta \backslash D_{n}}(b) \leq 1 / 2^{n+1}$. Let $S=\Delta \backslash \bigcup_{i=1}^{\infty} D_{i}$. By construction int $S=\varnothing$, and $u_{s}(b) \leq \Sigma_{i=1}^{\infty} u_{\Delta \backslash D_{i}}(b) \leq 1 / 2$, so that $(b, b) \in b_{r}\left(X_{S}\right) \backslash X_{s}$ by Theorem 1 .

Note. Theorem 1 also shows that one must be careful in the choice of $S$ to guarantee $b_{r}\left(X_{S}\right) \neq X_{S}$. Suppose, for example, that $S$ is a connected compact subset of $\Delta$ containing $\partial \Delta$, such that the only Jensen measures for $R(S)$ are point masses. Then $X_{S}$ is rationally convex, even when $R(S) \neq C(S)$. To see this, let $\left\{S_{n}\right\}_{n=1}^{\infty}$ be as in the definition of $u_{S}$.

Given $z \in S \backslash \partial \Delta$, let $\mu_{n}$ be harmonic measure for $z$ as a point of $S_{n}$; each $\mu_{n}$ is then a Jensen measure for $R\left(S_{n}\right)$. Each $\mu_{n}$ may be regarded as a measure on $\Delta$, and so, by passing to a subsequence, we may assume that $\left\{\mu_{n}\right\}$ converges weak-star on $\Delta$ to a measure $\mu$ with support contained in $S$. $\mu$ represents $z$ for $R(S)$ and is in fact a Jensen measure as we will now show.

Let $f \in R(S)$. Choose $f_{m}$ analytic on a neighborhood of $S$ so that $f_{m}$ converges to $f$ uniformly on $S$ as $m \rightarrow \infty$. If $n$ is large, $f_{m} \in R\left(S_{n}\right)$ and so

$$
\log \left|f_{m}(z)\right| \leq \int \log \left|f_{m}\right| d \mu_{n}
$$

Thus for any $\delta>0$ we have

$$
\log \left|f_{m}(z)\right| \leq \int \log \left(\left|f_{m}\right|+\delta\right) d \mu_{n}
$$

and so

$$
\log \left|f_{m}(z)\right| \leq \lim _{\delta ! 0} \lim _{n \rightarrow \infty} \int \log \left(\left|f_{m}\right|+\delta\right) d \mu_{n}=\int \log \left|f_{m}\right| d \mu
$$

Let $\epsilon>0$; then $\log \left(\left|f_{m}\right|+\epsilon\right)$ converges uniformly on $S$ to $\log (|f|+\epsilon)$ as $m \rightarrow \infty$, and so

$$
\lim _{m \rightarrow \infty} \int \log \left(\left|f_{m}\right|+\epsilon\right) d \mu=\int \log (|f|+\epsilon) d \mu
$$

On the other hand we have

$$
\begin{aligned}
\lim _{m \rightarrow \infty} \int \log \left(\left|f_{m}\right|+\epsilon\right) d \mu & \geq \underset{m \rightarrow \infty}{\lim } \int \log \left|f_{m}\right| d \mu \\
& \geq \lim _{m \rightarrow \infty} \log \left|f_{m}(z)\right|=\log |f(z)|
\end{aligned}
$$

Combining these results and applying monotone convergence we see that 


$$
\int \log |f| d \mu=\lim _{\epsilon \backslash 0} \int \log (|f|+\epsilon) d \mu \geq \log |f(z)|
$$

Thus $\mu$ is a Jensen measure for the point $z$ with respect to $R(S)$. By our assumption on $S, \mu$ is the unit point mass at $z$.

Choose $\chi \in C(\Delta)$ such that $0 \leq \chi \leq 1, \chi(z)=1$, and supp $\chi \subseteq$ int $\Delta$. Then

$$
\begin{aligned}
1 & \geq u_{S}(z)=\lim _{n \rightarrow \infty} u_{S_{n}}(z)=\lim _{n \rightarrow \infty} \int_{\partial S_{n} \backslash \partial \Delta} 1 d \mu_{n} \\
& \geq \lim _{n \rightarrow \infty} \int_{\partial S_{n}} \chi d \mu_{n}=\int_{S} \chi d \mu=\chi(z)=1 .
\end{aligned}
$$

Suppose now that $(z, w) \in h_{r}\left(X_{S}\right)$ and $z \in S \backslash \partial \Delta$. By Theorem 1 we have $u_{S}(w) \leq 1-$ $u_{s}(z)=0$. As noted earlier, $u_{s}(w)=0$ implies $w \in \partial \Delta$. Thus $b_{r}\left(X_{s}\right)=X_{s}$.

Note. It follows rather readily from Theorem 1 that if $b_{r}\left(X_{S}\right) \neq X_{S}$, then the 4-dimensional measure of $h_{r}\left(X_{S}\right) \backslash X_{S}$ is positive (in contrast to that of $X_{S}$ which, consisting of two copies of $S \times \partial \Delta$, has only positive 3-dimensional measure). To see this, extend $u_{s}$ to all of $\Delta$ by

$$
u_{S}(z)=1 \text { if } z \in \Delta \backslash S \text {. }
$$

Then $u_{S}$ is superharmonic on int $\Delta$ (see Lemma 6 below), so that the function $g$ defined on int $\Delta \times$ int $\Delta$ by $g(z, w)=u_{S}(z)+u_{S}(w)$ is superharmonic. If $\left(z_{0}, w_{0}\right) \epsilon$ $b_{r}\left(X_{s}\right) \backslash X_{s}$, then $\left(z_{0}, w_{0}\right) \in$ int $\Delta \times$ int $\Delta$ and, by Theorem $1, u_{s}\left(z_{0}\right)+u_{s}\left(w_{0}\right) \leq 1$. Thus if $B_{\epsilon}$ is any small ball around $\left(z_{0}, w_{0}\right)$ and if $\bar{m}$ denotes 4-dimensional Lebesgue measure on $C^{2}$ we have by the superharmonicity of $g$ :

$$
\frac{1}{\text { volume } B_{\epsilon}} \int_{B_{\epsilon}} g d \bar{m} \leq 1 .
$$

This implies that $\left\{(z, w) \in B_{\epsilon} \mid g(z, w)=u_{s}(z)+u_{s}(w) \leq 1\right\}$, which by Theorem 1 is contained in $b_{r}\left(X_{S}\right)$, has positive 4-dimensional measure.

A more careful analysis leads to a significant sharpening of this result; in fact, we are able to show that some of the "Gleason parts" for $R\left(X_{S}\right)$ have positive 4-dimensional measure. The concept of "Gleason part" has been useful in the study of analytic structure in the maximal ideal space of a uniform algebra (see, e.g., [3, Chapter VI]). If $T$ is a compact set in $\mathbf{C}^{n}$, the Gleason part (for the algebra $R(T)$ "=" $R\left(b_{r}(T)\right)$ ) of a point $p \in b_{r}(T)$ is the equivalence class of $p$ under the equivalence relation:

$$
p \sim q \text { if } \sup \left\{|f(p)-f(q)| \mid f \in R(T) \text { and } \sup _{T}|f| \leq 1\right\}<2 .
$$


(Thus, for instance, if $b_{r}(T)$ contains a connected analytic variety $V$, a normal family argument shows that the points of $V$ belong to a single part of $\left.b_{r}(T)_{0}\right)$

Theorem 2. If $b_{r}\left(X_{S}\right) \backslash X_{S} \neq \varnothing$, then there is a point $\left(z_{0}, w_{0}\right) \in b_{r}\left(X_{s}\right) \backslash X_{S}$ with $u_{s}\left(z_{0}\right)+u_{s}\left(w_{0}\right)<1$. Any such point belongs to a Gleason part of $b_{r}\left(X_{s}\right)$ with positive 4-dimensional measure.

The proof of Theorem 2 depends on several lemmas, for which we establish the following notation:

$m=2$-dimensional Lebesgue measure on the plane $\mathrm{C}$;

$S^{r}=\left\{z \in S \mid u_{S}(z) \leq r\right\}, 0<r \leq 1$ (note that $S^{r}$ is closed since by Lemma 6 below, $u_{S}$ is superharmonic).

Lemma 6. $u_{s}$ is superbarmonic on int $\Delta$ (as above, we consider $u_{s}$ extended to $\Delta$ by $u_{S} \equiv 1$ on $\left.\Delta \backslash S\right)$.

Proof. Take $\left\{S_{n}\right\}_{n=1}^{\infty}$ as in the definition of $u_{s}$. One verifies that each $u_{s_{n}}$ is superharmonic on int $\Delta$ by considering three cases:

(i) if $z \in \Delta \backslash S_{n}$, then $u_{S_{n}} \equiv 1$ near $z$;

(ii) if $z \in$ int $S_{n}$, then $u_{S}{ }_{n}$ is harmonic near $z$ by definition;

(iii) if $z \in \partial S_{n} \cap$ int $\Delta$, and $r<1-|z|$, then

$$
u_{S_{n}}(z)=1=\frac{1}{2 \pi} \int_{0}^{2 \pi} d \theta \geq \frac{1}{2 \pi} \int_{0}^{2 \pi} u_{S_{n}}\left(z+r e^{i \theta}\right) d \theta
$$

Thus $u_{s}$ is the pointwise limit on int $\Delta$ of the monotone increasing sequence of superharmonic functions $\left\{u_{s_{n}}\right\}$. So $u_{s}$ is superharmonic on int $\Delta$.

Corollary. If $0<u_{S}(z)<1$, there is a point $z^{\prime}$ near $z$ where $u_{S}\left(z^{\prime}\right)<u_{S}(z)$.

Proof. If $z \in$ int $S$, the statement is trivial since $u_{S}$ is harmonic near $z$. So suppose that $z \in \partial S \backslash \partial \Delta$. Then we can find $r$ arbitrarily small so that the set $E=\left\{\theta \mid z+r e^{i \theta} \in \Delta \backslash S\right\}$ has positive 1-dimensional measure. If we also had $u\left(z+r e^{i \theta}\right) \geq u(z)$ for all $\theta$, we would have

$$
\frac{1}{2 \pi} \int_{[0,2 \pi]} u\left(z+r e^{i \theta}\right) d \theta \geq \frac{1}{2 \pi} \int_{E} 1 d \theta+\frac{1}{2 \pi} \int_{[0,2 \pi] \backslash E} u(z) d \theta>u(z)
$$

This contradiction establishes that $u\left(z+r e^{i \theta}\right)<u(z)$ for some $\theta$.

This corollary verifies the easy half of Theorem 2, since $(z, w) \in b_{r}\left(X_{s}\right) \backslash X_{S}$ and $u_{s}(z)+u_{s}(w)=1$ imply $0<u_{s}(z)<1$. Applying the corollary to $z$, we have $z^{\prime}$ near $z$ with $u_{S}\left(z^{\prime}\right)<u_{S}(z)$. Then $\left(z^{\prime}, w\right)$ has the property that $u_{S}\left(z^{\prime}\right)+$ $u_{s}(w)<1$, and, of course, by Theorem $1\left(z^{\prime}, w\right) \in b_{r}\left(X_{s}\right) \backslash X_{s^{\circ}}$. 
Lemma 7. Fix $r, 0<r \leq 1$. If $0<u_{s}\left(z_{0}\right)<r$, then $z_{0}$ is not a peak point for $R\left(S^{r}\right)$.

Proof. Again take $\left\{S_{n}\right\}$ as in the definition of $u_{s}$. Since $u_{s_{n}}\left(z_{0}\right) \leq u_{s}\left(z_{0}\right)$ for all $n, z_{0} \in\left(S_{n}\right)^{r}$ for all $n$. Let $\mu_{n}$ be a probability measure supported on $\partial S_{n}^{r}$ such that for all functions $/$ harmonic in a neighborhood of $S_{n}^{r}$ we have

$$
\int_{\partial S_{n}^{r}} f d \mu_{n}=f\left(z_{0}\right)
$$

By passing to a subsequence we may assume that $\mu_{n}$ converges weak-star to a probability measure $\mu$ on $\partial S^{r}$ which then has the property that, for all $f$ harmonic in a neighborhood of $S^{r}, \int_{\partial S^{r}} f d \mu=f\left(z_{0}\right)$. It follows that $\mu$ represents $z_{0}$ for $R\left(S^{r}\right)$.

Choose $\chi \in C(\Delta)$ such that $0 \leq \chi \leq 1, \chi\left(z_{0}\right)=1$, and supp $\chi \subseteq$ int $\Delta$. Then

$$
\begin{aligned}
\int_{\partial S^{r}} \chi d \mu & =\lim _{n \rightarrow \infty} \int_{\partial S_{n}^{r}} \chi d \mu_{n} \leq \lim _{n \rightarrow \infty} \int_{\partial S_{n}^{r}} \frac{u_{S}}{r} d \mu_{n} \\
& =\lim _{n \rightarrow \infty} \frac{u_{S_{n}}\left(z_{0}\right)}{r}=\frac{u_{S}\left(z_{0}\right)}{r}<1
\end{aligned}
$$

where we have made use of the fact that $u_{S_{n}}=r$ on $\partial S_{n}^{r} \backslash \partial \Delta$ (if $u_{S_{n}}(z)<r$, then $u_{S}<r$ near $z$ ). So $\mu$ is not a point mass and therefore $z_{0}$ is not a peak point for $R\left(\hat{S}^{r}\right)$.

Lemma 8. Let $\left(z_{0}, w_{0}\right) \in b_{r}\left(X_{S}\right) \backslash\left(X_{s}\right)$, and suppose that $z_{0} \sim z_{1}$ in $s^{1-u_{s}\left(w_{0}\right)}$. Then $\left(z_{1}, w_{0}\right) \in b_{r}\left(X_{s}\right) \backslash X_{s}$, and $\left(z_{0}, w_{0}\right) \sim\left(z_{1}, w_{0}\right)$ in $b_{r}\left(X_{s}\right)$. Similarly, if $w_{1} \sim w_{0}$ in $S^{1-u_{S}\left(z_{1}\right)}$, then $\left(z_{1}, u_{1}\right) \in b_{r}\left(X_{S}\right) \backslash X_{S}$ and $\left(z_{1}, w_{1}\right) \sim\left(z_{1}, w_{0}\right)$ in $b_{r}\left(X_{s}\right)$.

Proof. Let

$$
\begin{aligned}
K & =b_{r}\left(X_{S}\right) \cap\left\{(z, w) \mid w=w_{0}\right\} \\
& =\left\{\left(z, w_{0}\right) \in \Delta^{2} \mid u_{S}(z) \leq 1-u_{S}\left(w_{0}\right)\right\}=S^{1-u_{S}\left(w_{0}\right)} \times\left\{w_{0}\right\} .
\end{aligned}
$$

$K$ is a compact subset of the plane $\left\{w=w_{0}\right\}$, and is nonempty since $\left(z_{0}, w_{0}\right) \in K$.

Since we have assumed that $z_{1} \in S^{1-u_{S}\left(w_{0}\right)}$, we have $u_{s}\left(z_{1}\right) \leq 1-u_{S}\left(w_{0}\right)$, so $\left(z_{1}, w_{0}\right) \in b_{r}\left(X_{S}\right)$, whence $\left(z_{1}, w_{0}\right) \in K$.

Let $F_{n} \in R\left(b_{r}\left(X_{S}\right)\right),\left|F_{n}\right| \leq 1$, and $F_{n}\left(z_{0}, w_{0}\right) \rightarrow+1$. Then $F_{n}\left(z, w_{0}\right) \in R(K)$, $\left|F_{n}\left(z, w_{0}\right)\right|^{n} \leq 1$, so by the assumption that $z_{0} \sim z_{1}$ in $S^{1-u S\left(w_{0}\right)}, F_{n}\left(z_{1}, w_{0}\right) \rightarrow+1$. 
Thus $\left(z_{0}, w_{0}\right) \sim\left(z_{1}, w_{0}\right)$ in $b_{r}\left(X_{S}\right)$. The second half of the lemma follows by applying the same argument with the roles of the coordinates reversed.

Proof of Theorem 2. Choose $\delta$ so that $0<\delta<1-u_{s}\left(z_{0}\right)-u_{s}\left(w_{0}\right)$. Let

$$
\begin{aligned}
& T_{1}=\left\{z \in S^{1-u_{S}\left(w_{0}\right)} \mid u_{S}(z) \leq u_{S}\left(z_{0}\right)+\delta, z \sim z_{0} \text { in } S^{1-u_{S}\left(w_{0}\right)}\right\}, \\
& T_{2}=\left\{w \in S^{1-u_{S}\left(z_{0}\right)-\delta} \mid w \sim w_{0} \text { in } S^{1-u_{S}\left(z_{0}\right)-\delta}\right\} .
\end{aligned}
$$

If $z \in T_{1},\left(z, w_{0}\right) \sim\left(z_{0}, w_{0}\right)$ in $h_{r}\left(X_{S}\right)$ by Lemma 8. We also have $u_{S}(z) \leq$ $u_{S}\left(z_{0}\right)+\delta$, so that $s^{1-u_{S}(z)} \supseteq s^{1-u_{s}\left(z_{0}\right)-\delta}$. Hence if $w \in T_{2}$, then $w \sim w_{0}$ in $s^{1-u S(z)}$. By Lemma $8,(z, w) \sim\left(z, w_{0}\right)$ in $b_{r}\left(X_{S}\right)$. Thus $(z, w) \sim\left(z_{0}, w_{0}\right)$ in $b_{r}\left(X_{S}\right)$, and so the part of $R\left(b_{r}\left(X_{S}\right)\right)$ to which $\left(z_{0}, w_{0}\right)$ belongs contains $T_{1} \times T_{2}$. Since $R\left(X_{S}\right)$ can be identified with $R\left(b_{r}\left(X_{S}\right)\right)$, the theorem will be proved if we show that $m\left(T_{1}\right)>0$ and $m\left(T_{2}\right)>0$.

For $0<R<1-\left|z_{0}\right|$, let

$$
E_{R}=\left\{z \in S \mid u_{S}(z)>u_{S}\left(z_{0}\right)+\delta\right\} \cap\left\{\left|z-z_{0}\right|<R\right\}
$$

By the superharmonicity of $u_{s}$, we have

$$
\begin{aligned}
u_{S}\left(z_{0}\right) & \geq \frac{1}{\pi R^{2}} \int_{\left|z-z_{0}\right|<R} u_{S}(z) d m \geq-\frac{1}{\pi R^{2}} \int_{E_{R}}\left(u_{S}\left(z_{0}\right)+\delta\right) d m \\
& =\left[u_{S}\left(z_{0}\right)+\delta \operatorname{lm}_{m}\left(E_{R}\right) / \pi R^{2} .\right.
\end{aligned}
$$

Thus $m\left(E_{R}\right) \leq \pi R^{2}\left(u_{s}\left(z_{0}\right) /\left(u_{s}\left(z_{0}\right)+\delta\right)\right)$. Note also that $u_{s}\left(z_{0}\right)<1-u_{s}\left(w_{0}\right)$, so by Lemma $7, z_{0}$ is not a peak point of $R\left(S^{1-u_{S}\left(w_{0}\right)}\right)$.

Let $K$ be a compact subset of $\mathbf{C}$, and suppose that $x \in K$ is not a peak point for $R(K)$. Given $\epsilon>0$, let

$$
P_{\epsilon}=\left\{y \in K \mid \sup \left\{|f(x)-f(y)|\left|f \in R(K), \sup _{K}\right| f \mid \leq 1\right\}<\epsilon\right\} .
$$

A the orem due to Browder says that

$$
\lim _{n \rightarrow \infty} \frac{m\left(P_{\epsilon} \cap\{y \in \mathbf{C}|| x-y \mid<1 / n\}\right)}{m(\{y \in \mathbf{C}|| x-y \mid<1 / n\})}=1
$$

(see [13], or [9, pp. 175-177]). In particular, if $\epsilon=1$ this says that the part of $R(K)$ to which $x$ belongs has density one at $x$. (This consequence of Browder's theorem was also proved by Melnikov [14] and by Wilken.) Applying this to $z_{0} \in S^{1-u_{S}\left(w_{0}\right)}$, we obtain 
$m\left(\left\{z \in S^{1-u_{S}\left(w_{0}\right)}|| z-z_{0} \mid<R, z \sim z_{0}\right.\right.$ in $\left.\left.S^{1-u_{S}\left(w_{0}\right)}\right\}\right)>\pi R^{2}\left(u_{S}\left(z_{0}\right) /\left(u_{S}\left(z_{0}\right)+\delta\right)\right)$ for $R$ sufficiently small.

Combining this result with the above we see that for $R$ small and positive:

$$
\begin{aligned}
m\left(T_{1}\right. & \left.\cap\left\{\left|z-z_{0}\right|<R\right\}\right) \\
= & m\left(\left\{z|| z-z_{0} \mid<R, u_{s}(z) \leq u_{s}^{\prime}\left(z_{0}\right)+\delta, z \sim z_{0} \text { in } s^{1-u_{S}\left(w_{0}\right)}\right\}\right) \\
= & m\left(\left\{\left|z-z_{0}\right|<R\right\}\right)-m\left(E_{R}\right) \\
& -m\left(\left\{z|| z-z_{0} \mid<R, u_{S}(z) \leq u_{s}\left(z_{0}\right)+\delta, \text { but not } z \sim z_{0} \text { in } S^{1-u_{S}\left(w_{0}\right)}\right\}\right) \\
> & \pi R^{2}-\pi R^{2}\left(\frac{u_{s}\left(z_{0}\right)}{u_{S}\left(z_{0}\right)+\delta}\right)-\left[\pi R^{2}-\pi R^{2}\left(\frac{u_{S}\left(z_{0}\right)}{u_{S}\left(z_{0}\right)+\delta}\right)\right]=0 .
\end{aligned}
$$

In particular, $m\left(T_{1}\right)>0$.

Finally, $u_{s}\left(w_{0}\right)<1-u_{s}\left(z_{0}\right)-\delta$ by definition of $\delta$, so by Lemma $7 w_{0}$ is not a peak point for $R\left(S^{1-u_{S}\left(z_{0}\right)-\delta}\right)$. Thus $m\left(T_{2}\right)>0$ since nontrivial parts have positive measure. This and the last corollary complete the proof of Theorem 2.

2. Let $B$ denote the closed unit ball in $\mathrm{C}^{2}=\left\{\left.(z, w)|| z\right|^{2}+|w|^{2} \leq 1\right\}$. If $T$ is an arbitrary compact subset of the open unit disk, we associate with $T$ a subset of the 3-sphere $\left\{|z|^{2}+|w|^{2}=1\right\}$ by defining

$$
\tilde{X}_{T}=\{(z, w) \in \partial B \mid z \in T\}
$$

Two observations about $R\left(\widetilde{X}_{T}\right)$ can be made immediately. First, since it is well known that if $X$ is a compact subset of $\mathrm{C}^{n}$ then $R(X)$ is generated by $n+1$ functions (for a proof see Lemma 11), the algebra $R\left(\widetilde{X}_{T}\right)$ is finitely generated. Secondly, each point of $\tilde{X}_{T}$ is a peak point for $R\left(\tilde{X}_{T}\right)$; this is in itself a trivial observation, since if $Y$ is any compact subset of $\partial B$ then each point $\left(z_{0}, w_{0}\right) \in Y$ is a peak point for $P(Y)$-hence for $R(Y)$-because $P(z, w)=\left(\bar{z}_{0} z+\bar{w}_{0} w+1\right) / 2$ peaks at $\left(z_{0}, w_{0}\right)$. (This explicit peaking function was pointed out to me by several people.) This latter fact, however, becomes particularly significant in conjunction with the following result:

Theorem 3. Suppose that the only Jensen measures for $R(T)$ are trivial, and $R(T) \neq C(T)$. Then

(i) the maximal ideal space of $R\left(\tilde{X}_{T}\right)$ is $\tilde{X}_{T}$, i.e., $\widetilde{X}_{T}$ is rationally convex;

(ii) $R\left(\tilde{X}_{T}\right) \neq C\left(\tilde{X}_{T}\right)$. 
Thus for such a $T$ properties I, II, III mentioned in the introduction are satisfied by the algebra $A=R\left(\widetilde{X}_{T}\right)$. The proof of Theorem 3 can be divided into two lemmas.

Lemma 9. Suppose that $T$ is a compact subset of the open unit disk, and the only Jensen measures for $R(T)$ are trivial. Then $\tilde{X}_{T}$ is rationally convex.

Proof. Suppose $(\alpha, \beta) \in b_{r}\left(\tilde{X}_{T}\right)$. Let $\mu$ be a Jensen measure on $\tilde{X}_{T}$ for the homomorphism corresponding to evaluation at $(\alpha, \beta)$. We assert that

$$
\operatorname{supp} \mu \subseteq\left\{(z, w) \in \tilde{X}_{T} \mid z=\alpha\right\}
$$

The latter set is a circle which we denote by $\Gamma_{\alpha}$.

Observe that $\alpha \in T$, since otherwise $z-\alpha$ vanishes at $(\alpha, \beta)$ but has no zero on $\tilde{X}_{T}$. Define a measure $\bar{\mu}$ on $T$ by

$$
\int_{T} g d \bar{\mu}=\int_{\tilde{X}_{T}} g(z) d \mu(z, w), \quad g \in C(T) .
$$

Let $f \in R(T)$; then since $\mu$ is a Jensen measure for $(\alpha, \beta)$

$$
\log |f(a)| \leq \int_{\tilde{X}_{T}} \log |f(z)| d \mu(z, w)=\int_{T} \log |f(z)| d \bar{\mu}(z)
$$

So $\bar{\mu}$ is a Jensen measure for $\alpha$ with respect to $R(T)$. By assumption, $\bar{\mu}$ is the unit point mass at $\alpha$. Hence

$$
\mu\left(\Gamma_{\alpha}\right)=\int_{\Gamma_{a}} d \mu=\int_{\{a\}} d \bar{\mu}=1
$$

Since $\mu$ is a probability measure, $\operatorname{supp} \mu \subseteq \Gamma_{a}$.

Now $w \neq 0$ on $\widetilde{X}_{T}$, so $1 / w \in R\left(\widetilde{X}_{T}\right)$. Thus

$$
\left|\frac{1}{\beta}\right|=\left|\int_{\tilde{X}_{T}} \frac{1}{w} d \mu\right| \leq \int_{\Gamma_{\alpha}} \frac{1}{|w|} d \mu=\frac{1}{\left(1-|\alpha|^{2}\right)^{1 / 2}}
$$

or $|\beta| \geq\left(1-|\alpha|^{2}\right)^{1 / 2}$. Since $(\alpha, \beta) \in B$ we must have $|\beta|=\left(1-|\alpha|^{2}\right)^{1 / 2}$, whence $(\alpha, \beta) \in \tilde{X}_{T^{\bullet}}$

Lemma 10. Let $T$ be a compact subset of the open disk sucb that $R(T) \neq C(T)$. Then $R\left(\tilde{X}_{T}\right) \neq C\left(\tilde{X}_{T}\right)$.

Proof. Let $\mu \in C(T)^{*}, \mu \perp R(T), \mu \neq 0$. If $z_{0} \in$ int $\Delta$ let $m_{z_{0}}$ be normalized Lebesgue measure on the circle $\Gamma_{z_{0}}=\left\{\left(z_{0}, w\right)|| w \mid=\left(1-\left|z_{0}\right|^{2}\right)^{1 / 2}\right\}$. Define a measure $\bar{\mu}$ on $\tilde{X}_{T}$ by 


$$
\int \tilde{X}_{T} g d \bar{\mu}=\int_{T}\left[\int_{\Gamma_{z}} g(z, w) d m_{z}(w)\right] d \mu(z), \quad g \in C\left(\tilde{X}_{T}\right)
$$

Note that $\bar{\mu} \neq 0$. We wish to show that $\bar{\mu} \perp R\left(\tilde{X}_{T}\right)$, so let $f(z, w)$ be a rational function holomorphic in a neighborhood of $\tilde{X}_{T}$. For $z$ near $T$ in $\mathrm{C}$ we may define a function

$$
a(z)=\int_{\Gamma_{\boldsymbol{z}}} f(z, w) d m_{\boldsymbol{z}}(w)
$$

we assert that $a(z)$ is analytic in a neighborhood of $T$.

Fix $z_{0} \epsilon T$. For some $\epsilon>0$ and $R_{1}>\left(1-\left|z_{0}\right|^{2}\right)^{1 / 2}>R_{2}>0, f(z, w)$ is analytic on

$$
\Omega=\left\{(z, w) \in \mathbf{C}^{2}|| z-z_{0}\left|<\epsilon, R_{1}>\right| w \mid>R_{2}\right\}
$$

On $\Omega, f(z, w)$ may be expanded in a Laurent series in $w$ :

$$
f(z, w)=\sum_{n=-\infty}^{\infty} a_{n}(z) w^{n}
$$

where

$$
a_{n}(z)=\frac{1}{2 \pi i} \int_{|w|=\left(1-\left|z_{0}\right|^{2}\right)^{1 / 2}} \frac{f(z, w) d w}{w^{n+1}}
$$

is analytic in $z$. But then

$$
a(z)=\int_{\Gamma_{z}} f(z, w) d m_{z}(w)=\int_{\Gamma_{z}} \sum_{n=-\infty}^{\infty} a_{n}(z) w^{n} d m_{z}(w)=a_{0}(z)
$$

is analytic for $z$ near $z_{0}$. So the assertion is proven.

Consequently we have

$$
\int \tilde{x}_{T} f d \bar{\mu}=\int_{T}\left[\int_{\Gamma_{z}} f(z, w) d m_{z}(w)\right] d \mu(z)=\int_{T} a(z) d \mu(z)=0 .
$$

Thus $R\left(\widetilde{X}_{T}\right) \notin C\left(\tilde{X}_{T}\right)$. This completes the proof of Theorem 3 .

3. We wish to consider sets of the type $\tilde{X}_{T}$ described in $\$ 2$, where we again require that $T$ be a compact subset of int $\Delta$. We impose the additional requirement that $T$ be a "Swiss cheese", so that $R(T) \neq C(T)$. For our purposes a Swiss 
cheese $T$ is a compact subset of $\mathbf{C}$ with empty interior, formed by deleting from a piecewise smoothly bounded connected compact set $S$ a sequence of nonempty piecewise smoothly bounded simply connected open sets $O_{m}$ subject to the conditions:

(i) $\bar{O}_{m} \cap \bar{O}_{n}=\varnothing$ if $m \neq n$;

(ii) $\bar{O}_{m}^{m} \subseteq$ int $S$ for all $m$;

(iii) $\Sigma_{m=1}^{\infty}$ length $\left(\partial O_{m}\right)<\infty$.

With this definition the example in Browder's book [9, p. 192] is a Swiss cheese.

Theorem 4. Let $T \subseteq$ int $\Delta$ be a Swiss cheese. Let $K$ be a compact subset of $\widetilde{X}_{T}$ with nonempty interior in the topology of $\widetilde{X}_{T^{*}}$. Then $R(K) \neq C(K)$. Consequently, the restriction of $R\left(\widetilde{X}_{T}\right)$ to $K$ is not dense in $C(K)$. (Note that, in particular, $T$ may be chosen so that $\tilde{X}_{T}$ satisfies Theorem 3.)

Proof. We will construct a measure $\mu$ on $K$ such that $\mu \perp R(K)$ and $\mu \neq 0$. For simplicity we may suppose that $(0,1)$ is in the interior of $K$ relative to $\widetilde{X}_{T}$. It follows that, for some positive $\epsilon<1$ and some positive $\theta_{0}<\pi, K$ contains the set $F \cap \widetilde{X}_{T}$ where

$$
F=\left\{(z, w) \in \partial B|| z|\leq \epsilon,| \arg w \mid \leq \theta_{0}\right\}
$$

Since $T$ is a Swiss cheese, we may write $T=S \backslash \bigcup_{m=1}^{\infty} O_{m}$ with $S$ and $O_{m}$ as in the above definition. Let

$$
K_{n}=\left\{(z, w) \in F \mid z \in S \backslash \bigcup_{m=1}^{n} O_{m}\right\}, \text { if } n>0
$$

so that $K_{n+1} \subseteq K_{n}$ for all $n>0$, and $\bigcap_{n=1}^{\infty} K_{n}=K \cap F$. If $U$ is any small open neighborhood of

$$
\left\{(z, \theta) \in \mathbf{C} \times R|| z|\leq \epsilon,| \theta \mid \leq \theta_{0}\right\}
$$

then the map $\psi: U \rightarrow \partial B$ defined by

$$
\psi(z, \theta)=\left(z, e^{i \theta}\left(1-|z|^{2}\right)^{1 / 2}\right), \quad(z, \theta) \in U
$$

establishes a real analytic diffeomorphism of $U$ with an open neighborhood of $K_{1}$ as a subset of $\partial B$. For $n=1,2, \cdots$ let $\partial_{0} K_{n}$ denote the boundary of $K_{n}$ relative to $\partial B$.

We may visualize $K_{1}, K_{2}, \cdots$ and $\partial_{0} K_{1}, \partial_{0} K_{2}, \cdots$ by means of the map $\psi^{-1}$ : 


$$
\begin{aligned}
\psi^{-1}\left(K_{n}\right)= & \left\{z \in S \backslash \bigcup_{m=1}^{n} o_{m}|| z \mid \leq \epsilon\right\} \times\left\{|\theta| \leq \theta_{0}\right\}, \quad n>0 ; \\
\psi^{-1}\left(\partial_{0} K_{n}\right)= & \left\{z \in \partial S \cup\left(\bigcup_{m=1}^{n} \partial_{m}\right)|| z \mid \leq \epsilon\right\} \times\left\{|\theta| \leq \theta_{0}\right\} \\
& \cup\left\{z \in S \backslash \bigcup_{m=1}^{n} O_{m}|| z \mid=\epsilon\right\} \times\left\{|\theta| \leq \theta_{0}\right\} \\
& \cup\left\{z \in S \backslash \bigcup_{m=1}^{n} O_{m}|| z \mid \leq \epsilon\right\} \times\left\{-\theta_{0}, \theta_{0}\right\}, \quad n>0 .
\end{aligned}
$$

From this it is evident that, for each $n, n=1,2, \cdots$, the set $K_{n}$ is the closure of an open subset $V_{n}$ of $\partial B$ and

(i) $\partial_{0} K_{n}$ is "piecewise smooth";

(ii) $V_{n}$ "lies on one side of $\partial_{0} K_{n} "$.

We may therefore apply Stokes' the orem to $V_{n}$ and $\partial_{0} K_{n}$. (See [15, pp. 271-275] for precise definitions and a statement of an appropriate form of Stokes' theorem.)

Let $f$ be analytic in a neighborhood of $K_{n}$. The preceding remarks show that

$$
\begin{aligned}
\int_{\partial_{0} K_{n}} f d z & \wedge d w \\
& =\int_{V_{n}}\left(\frac{\partial f}{\partial \bar{z}} d \bar{z}+\frac{\partial f}{\partial \bar{w}} d \bar{w}\right) \wedge d z \wedge d w=0
\end{aligned}
$$

Let $\mu_{n}$ denote the measure on $\partial_{0} K_{n}$ corresponding to the form $d z \wedge d w$. We may regard $\left\{\mu_{n}\right\}$ as a sequence of measures on $K_{1}$, because $K_{n} \subseteq K_{1}$ for all $n>0$. We assert that the condition

$$
\sum_{m=1}^{\infty} \text { length }\left(\partial O_{m}\right)<\infty
$$

implies that the $\mu_{n}$ converge in norm. In fact, suppose that $f$ is $C^{\infty}$ in a neighborhood of $K_{1}$ and $\sup _{K_{1}}|f| \leq 1$. If $N>M>0$, then 


$$
\begin{aligned}
& \left|\int_{K_{1}} f d \mu_{N}-\int_{K_{1}} f d \mu_{M}\right|=\left|\int_{\partial_{0} K_{N}} f(z, w) d z \wedge d w-\int_{\partial_{0} K_{M}} f(z, w) d z \wedge d w\right| \\
& =\left|\int_{\psi^{-1}\left(\partial_{0} K_{N}\right)} f(\psi(z, \theta)) d(z \circ \psi) \wedge d(w \circ \psi)-\int_{\psi^{-1}\left(\partial_{0} K_{\|}\right)} f(\psi(z, \theta)) d(z \circ \psi) \wedge d(w \circ \psi)\right| \\
& =\mid \sum_{m=M+1}^{N}\left(\int_{\left\{(z, \theta)|| z\left|\leq \epsilon, z \epsilon \partial O_{m},\right| \theta \mid \leq \theta_{0}\right\}} f\left(z, e^{i \theta}\left(1-|z|^{2}\right)^{1 / 2}\right) d z \wedge\left(\frac{\partial w}{\partial \bar{z}} d \bar{z}+\frac{\partial w}{\partial \theta} d \theta\right)\right. \\
& -\int_{\left\{(z, \theta)|| z\left|=\epsilon, z \in O_{m},\right| \theta \mid \leq \theta_{0}\right\}} /\left(z, e^{i \theta}\left(1-|z|^{2}\right)^{1 / 2}\right) d z \wedge\left(\frac{\partial w}{\partial \bar{z}} d \bar{z}+\frac{\partial w}{\partial \theta} d \theta\right) \\
& \left.-\int_{\left\{(z, \theta)|| z \mid \leq \epsilon, z \epsilon O_{m}, \theta= \pm \theta_{0}\right\}} f\left(z, e^{i \theta}\left(1-|z|^{2}\right)^{1 / 2}\right) d z \wedge\left(\frac{\partial w}{\partial \bar{z}} d \bar{z}+\frac{\partial w}{\partial \bar{\theta}} d \theta\right)\right) \mid \\
& \leq \sum_{m=M+1}^{N}\left(\mid \int_{\left\{|z| \leq \epsilon, z \epsilon \partial O O_{m},|\theta| \leq \theta_{0}\right\}} f\left(z, e^{\left.i \theta\left(1-|z|^{2}\right)^{1 / 2}\right) d z \wedge\left(i e^{i \theta}\left(1-|z|^{2}\right)^{1 / 2} d \theta\right)} \mid\right.\right. \\
& +\mid \int_{\left\{|z|=\epsilon, z \in O_{m},|\theta| \leq \theta_{0}\right\}}\left(\left(z, e^{i \theta}\left(1-|z|^{2}\right)^{1 / 2}\right) d z \wedge\left(i e^{i \theta}\left(1-|z|^{2}\right)^{1 / 2} d \theta\right) \mid\right. \\
& \left.+\left|\int_{\left\{|z| \leq \epsilon, z \in O_{m}, \theta= \pm \theta_{0}\right\}} f\left(z, e^{i \theta}\left(1-|z|^{2}\right)^{1 / 2}\right) d z \wedge\left(\frac{-e^{i \theta} z}{2\left(1-|z|^{2}\right)^{1 / 2}} d \bar{z}\right)\right|\right) \\
& \leq \sum_{m=M+1}^{N}\left(\sup _{K_{1}}|/| \cdot \operatorname{length}\left(\partial O_{m}\right) \cdot 2 \theta_{0}+\underset{K_{1}}{\sup }|/| \cdot \operatorname{length}\left(\partial O_{m}\right) \cdot 2 \theta_{0}\right. \\
& \left.+2 \sup _{K_{1}}|f| \cdot 2 \operatorname{area}\left(O_{m}\right) \cdot \frac{\epsilon}{2\left(1-\epsilon^{2}\right)^{1 / 2}}\right) \\
& \leq \sum_{m=11+1}^{\mathrm{V}}\left(4 \theta_{0} \text { length }\left(\lambda O_{m}\right)+\frac{2 \epsilon}{\left(1-\epsilon^{2}\right)^{T / 2}} \operatorname{area}\left(O_{m}\right)\right)
\end{aligned}
$$

which by (2) converges to zero independent of $f$ as $M, N \rightarrow \infty$.

Let $\mu$ be the uniform limit of the $\mu_{n}$. Since $\operatorname{supp} \mu_{n} \subseteq K_{n}$ and $K_{n+1} \subseteq K_{n}$, we have supp $\mu \subseteq \cap_{n=1}^{\infty} K_{n}=K \cap F$. If $f$ is analytic in a neighborhood of $K$, then $f$ is analytic in a neighborhood of some $K_{M}$, and for $N \geq M$ we have $\int_{\partial_{0}} K_{N} f d \mu_{N}=0$, by (1). So

$$
\int_{K} f d \mu=\lim _{N \rightarrow \infty} \int_{\partial_{0} K_{N}} f d \mu_{N}=0
$$

Thus $\mu \perp R(K)$, and it remains only to show that $\mu \neq 0$.

Choose $M>0$ so that $O_{M} \cap\{|z|<\epsilon\} \neq \varnothing$. Let $g(z)$ be a bounded Borel function on $C$ such that $g(z)=0$ unless $z \in\{|z| \leq \epsilon\} \cap \partial O_{M}$, and such that

$$
\left.\int_{\{|z| \leq \epsilon, z \in \partial O}\right\}{ }_{M} g(z) d z=1
$$


Let $f(z, w)=g(z) / i w$; then for $N \geq M, \mu_{N}$ agrees with $\mu_{M}$ on the support of $f$, so

$$
\begin{aligned}
& \int_{K_{1}} f d \mu_{N}=\int_{K_{1}} f d \mu_{M}=\int_{\partial_{0} K_{M}} \frac{g(z)}{i w} d z \wedge d w \\
& \left.=\int_{\{|z| \leq \epsilon, z \in \partial O},|\theta| \leq \theta_{0}\right\} \frac{g(z)}{i e^{i \theta}\left(1-|z|^{2}\right)^{1 / 2}} d z \wedge\left(i e^{i \theta}\left(1-|z|^{2}\right)^{1 / 2} d \theta\right) \\
& =2 \theta_{0} \int_{\left\{|z| \leq \epsilon, z \in \partial O_{M}\right\}} g(z) d z=2 \theta_{0} \neq 0 \text {. }
\end{aligned}
$$

Thus $\int_{K} f d \mu \neq 0$, and so $\mu \neq 0$.

4. Our final objective is the construction of smooth sets with the pathological behavior discussed in the first two sections. The key result we need is that for any compact subset $X$ of $\mathrm{C}^{2}$ we can find a smooth function $f$ such that $z, w, f$ generate $R(X)$. More precisely, we have the following result:

Lemma 11. Let $X$ be a compact subset of $\mathbf{C}^{n}$. There is an $f \in C_{0}^{\infty}\left(\mathbf{C}^{n}\right)$ such that the restrictions to $X$ of $z_{1}, \cdots, z_{n}$ and $f$ generate $R(X)$. Furthermore, $f$ may be chosen so that $\left.f\right|_{b_{r}(X)} \in R\left(b_{r}(X)\right)$, i.e., $f_{b_{r}(X)}$ coincides with the natural extension of $\left.f\right|_{X}$ to the maximal ideal space of $R(X), b_{r}(X)$.

As the proof is simply a modification of the proof that $R(X)$ has $n+1$ generators (see, e.g., $[9$, p. 16]), we leave it to be included as an appendix.

One addition to the notation previously introduced is needed.

$\rho_{X}$, for $X$ a compact subset of $\partial B$, will denote an arbitrarily chosen $C^{\infty}$ realvalued function on $\partial B$ which is zero precisely on $X$. It is easy to construct such a function. A similar function was used by Freeman in Example 5.3 in [16].

Lemma 12. Let $X$ be a compact subset of $\partial B$. Let It be a uniform algebra on $X$ which contains the polynomials in $z$ and $w$, and suppose that there is a function $f \in C^{\infty}(\partial B)$ sucb that $z, w, f$ generate $\mathcal{X}$. Define a uniform algebra $\mathfrak{U}^{\prime}$ on $\partial B$ by

$$
\mathfrak{U}^{\prime}=\left\{g \in C(\partial B)|g|_{X} \in \mathfrak{U}\right\} .
$$

Let $\Phi: \partial B \rightarrow \mathrm{C}^{6}$ by

$$
\Phi(z, w)=\left(z, w, f(z, w), \rho_{X}(z, w), \bar{z} \rho_{X}(z, w), \bar{w} \rho_{X}(z, w)\right)
$$

Then $\Phi$ establishes an isometric isomorphism between the algebras $\mathscr{U}^{\prime}$ and $P[\Phi(\partial B)]$ by $F \leftrightarrow F \circ \Phi^{-1}$ for $F \in \mathfrak{U}^{\prime}$.

Proof. First observe that $z, w, f, \rho_{X}, \bar{z} \rho_{X}$ and $\vec{w} \rho_{X}$ are in $\mathcal{X}^{\prime}$. In fact, we claim that they generate $\mathscr{U}^{\prime}$. Let $\mathfrak{B}$ be the subalgebra of $\mathfrak{X}^{\prime}$ generated by these 
functions, and let $\mu$ be any measure on $\partial B$ orthogonal to $\Re$. We will show that $\mathfrak{U}^{\prime}=\mathfrak{B}$ by showing that $\mu \perp \mathfrak{U}^{\prime}$.

Now if $g \in C(\partial B)$ and $\left.g\right|_{X} \equiv 0$, then $g$ is uniformly approximable on $\partial B$ by polynomials in $z, w, \rho_{X}, \bar{z} \rho_{X}, \bar{w} \rho_{X}$-this follows from the Stone-Weierstrass theorem for locally compact spaces, since the real-valued functions $\rho_{X}(z+\bar{z})$, $\rho_{X}(z-\bar{z}), \rho_{X}(w+\bar{w})$ and $\rho_{X}(w-\bar{w})$ separate the points of $\partial B \backslash X$. Thus supp $\mu \subseteq X$. But $B$ contains all polynomials in $z, w$ and $f$, so by the hypothesis that $z, w$ and $f$ generate $\mathfrak{U}$, we have $\mu \perp \mathfrak{U}^{\prime}$.

Since the coordinates of the map $\Phi$ generate $\mathcal{U}^{\prime}$, it follows that $\Phi$ establishes the required isomorphism.

Theorem 5. There is a $C^{\infty}$ 3-sphere $\Sigma_{1} \subseteq C^{6}$ such that $b\left(\Sigma_{1}\right) \backslash \Sigma_{1}$ is nonempty but does not contain analytic structure.

Proof. Let $Y=X_{S}$ with $S$ chosen as in Wermer [7] or as in the example on p.365 with $b=0$. $Y$ has the following relevant properties:

(i) $(0,0) \in b_{r}(Y)$;

(ii) $b_{r}(Y)$ does not contain analytic structure;

(iii) $Y \subseteq \partial \Delta^{2}$, the topological boundary of the unit bicylinder.

By Lemma 11 there is an $f \in C^{\infty}\left(C^{2}\right)$ such that $z, w$ and $f$ generate $R(Y)$, and $f \in R\left(b_{r}(Y)\right)$.

Let $X=b_{r}(Y) \cap \partial B, \mathscr{U}=$ the uniform algebra on $X$ generated by $z, w, f$.

We observe as follows that the maximal ideal space of $\mathscr{A}$, which we shall denote by $M$, may be identified with $h_{r}(Y) \cap B$.

If $\left(z_{0}, w_{0}\right) \in h_{r}(Y) \cap B$, and $G$ is a polynomial in $z, w, f$, then

$$
\begin{aligned}
\left|G\left(z_{0}, w_{0}\right)\right| & \leq \max \left\{|G(z, w)| \mid(z, w) \in\left[(\text { Silov boundary } R(Y)) \cap\left(B \cap b_{r}(Y)\right)\right]\right. \\
\left.\cup\left(\partial B \cap b_{r}(Y)\right)\right\} & \\
& =\max _{X}|G|
\end{aligned}
$$

by the Rossi local maximum modulus principle. Thus evaluation at $\left(z_{0}, w_{0}\right)$ induces a homomorphism on $\mathfrak{A}$.

If, on the other hand, $m$ is a homomorphism on $\mathcal{U}$, then since $z, w, f$ generate $R(Y), m$ induces a homomorphism of $R(Y)$ and so corresponds to evaluation of $z, w, f$ at some point $\left(z_{0}, w_{0}\right) \in b_{r}(Y)$. Since $B$ is polynomially convex, we must have $\left(z_{0}, w_{0}\right) \in b_{r}(Y) \cap B$.

We have therefore established the following facts about the maximal ideal space of $\mathcal{U}$ : 
(i) $(0,0) \in M$;

(ii) $M$ does not contain analytic structure.

Let $\mathfrak{U}^{\prime}=\left\{F \in C(\partial B)|F|_{X} \in \mathfrak{U}\right\}$. The maximal ideal space of $\mathfrak{U}^{\prime}$ may evidently by identified with $\partial B \cup M$, and the Silov boundary of $\mathscr{U}^{\prime}$ is $\partial B$. Clearly the maximal ideal space of $\mathcal{U}^{\prime}$ does not contain analytic structure.

Let $\Phi$ be the map of $\left(z, w, f, \rho_{X}, \bar{z} \rho_{X}, \bar{w} \rho_{X}\right)$ as described in Lemma 12. Let $\Sigma_{1}=\Phi(\partial B)$. By Lemma 12, $\mathfrak{U}^{\prime}$ and $P\left(\Sigma_{1}\right)$ are equivalent uniform algebras, so $b\left(\Sigma_{1}\right) \backslash \Sigma_{1}$ is nonempty but $\Sigma_{1}$ does not contain analytic structure.

Note. The same technique may be applied to Stolzenberg's example [8] to obtain such a $\Sigma_{1}$ in $\mathbf{C}^{5}$ rather than $\mathbf{C}^{6}$.

Theorem 6. There is a polynomially convex $C^{\infty}$ 3-sphere $\Sigma_{2} \subseteq \mathrm{C}^{6}$ such that every point of $\Sigma_{2}$ is a peak point for $P\left(\Sigma_{2}\right)$, but $P\left(\Sigma_{2}\right) \neq C\left(\Sigma_{2}\right)$.

Proof. Let $X$ be the set $\tilde{X}_{T}$ of The orem 3. Since $X \subseteq \partial B$, by Lemma 11 there is an $f \in C^{\infty}(\partial B)$ such that $z, w$ and $f$ generate $R(X)$. Let

$$
\mathfrak{U}=R(X), \quad \mathfrak{U}^{\prime}=\left\{F \in C(\partial B)|F|_{X} \in \mathfrak{U}\right\} .
$$

Clearly the maximal ideal space of ${ }^{\prime}{ }^{\prime}$ is $\partial B$, every point of $\partial B$ is a peak point for $\mathfrak{U}^{\prime}$, but $\mathfrak{U}^{\prime} \neq C(\partial B)$. Let $\Phi$ be the corresponding map as described in Lemma 12 , and let $\Sigma_{2}=\Phi(\partial B)$. Then $\mathfrak{U}^{\prime}$ and $P\left(\Sigma_{2}\right)$ are equivalent uniform algebras, so $\Sigma_{2}$ has the required properties.

\section{APPENDIX (PROOF OF LEMMA 11)}

Notation. If $a=\left(\alpha_{1}, \cdots, \alpha_{2 n}\right)$ with each $\alpha_{i}$ a nonnegative integer, then $D^{a}$ is the differential operator on $C_{0}^{\infty}\left(C^{n}\right)$ defined by

$$
D^{a} f=\frac{\partial^{a} \mid}{\partial^{a}{ }^{1} z_{1} \ldots \partial^{a}{ }^{n} z_{n} \partial^{a}{ }^{n+1} \bar{z}_{1} \ldots \partial^{a} 2 n \bar{z}_{n}}, \quad f \in C_{0}^{\infty}\left(\mathbf{C}^{n}\right) \text {, }
$$

where $|a|=\sum_{i=1}^{2 n} a_{i}$

Lemma 11. Let $X$ be a compact subset of $\mathbf{C}^{n}$. There is an $f \in C_{0}^{\infty}\left(\mathbf{C}^{n}\right)$ sucb that the restrictions to $X$ of $z_{1}, \cdots, z_{n}$ and $f$ generate $R(X)$. Furthermore, $f$ may be chosen so that $f_{h_{r}(X)} \in R\left(h_{r}(X)\right)$, i.e., $f_{h_{r}(X)}$ coincides with the natural extension of $\left.f\right|_{X}$ to $\vec{b}_{r}^{r}(X)$.

Proof of lemma. Choose a sequence of polynomials $g_{1}, g_{2}, \cdots$ so that each $g_{m}$ has no zero on $X$, and so that $\left\{p / g_{m} \mid p\right.$ is a polynomial, $\left.1 \leq m<\infty\right\}$ is dense in $R(X)$. For each $m$ let $b_{m}$ be a $C_{0}^{\infty}$-extension of $1 / g_{m}$ from $b_{r}(X)$ to $C^{n}$ (note 
that $g_{m}$ does not vanish on $\left.b_{r}(X)\right)$, and arrange so that the supports of the $b_{m}$ 's are all contained in some fixed compact subset of $\mathrm{C}^{n}$.

Now choose positive constants $c_{1}, c_{2}, \cdots$ so that

(i) $c_{m} \sup _{x \in \mathrm{C}^{n}} \sup _{0} \leq|a| \leq m\left|D^{a} b_{m}(x)\right|<2^{-m}$,

(ii) for $1 \leq k<m, c_{m} \sup _{x \in X}\left|g_{k}(x) / g_{m}(x)\right|<2^{-m} c_{k}$, for $m=1,2, \cdots$. Let $f=\Sigma_{k=1}^{\infty} c_{k} b_{k}$. By (i), $f \in C_{0}^{\infty}\left(\mathbf{C}^{n}\right)$, and $\left.f\right|_{b_{r}(X)} \in R\left(b_{r}(X)\right)$.

The proof may now be concluded exactly as in [9]; for this purpose we henceforth regard all functions as functions on $X$, so that for example $b_{k}=1 / g_{k}$. We will show that the algebra $A$ generated by $z_{1}, z_{2}, \cdots, z_{n}, f$ is $R(X)$. By choice of $\left\{g_{m}\right\}$, is it sufficient to show that $1 / g_{k} \in A$ for all $k$.

Let $f_{k}=\Sigma_{m=k}^{\infty} c_{m} / g_{m}, k=1,2, \cdots$. Then $f_{1}=f \in A$, and if $1 / g_{1}, 1 / g_{2}$, $\cdots, 1 / g_{k-1} \in A$, then $f_{k} \in A$. Proceed by induction: if $f_{k} \in A$, then $f_{k} g_{k} \in A$, and $f_{k} g_{k}=c_{k}+\Sigma_{m=k+1}^{\infty} c_{m} g_{k} / g_{m}$. By (ii), $c_{k}>\sup _{X}\left|\Sigma_{m=k+1}^{\infty} c_{m} g_{k} / g_{m}\right|$, so $\left(f_{k} g_{k}\right)^{-1} \in A$, so $1 / g_{k} \in A$. Thus the induction is complete and so is the proof of the lemma.

\section{BIBLIOGRAPHY}

1. J. Wermer, Polynomial approximation on an arc in $\mathrm{C}^{3}$, Ann. of Math. (2) 62 (1955), 269-270. MR 17, 255.

2. W. Rudin, Subalgebras of spaces of continuous functions, Proc. Amer. Math. Soc. 7 (1956), 825-830. MR 18, 587.

3. T. Gamelin, Uniform algebras, Prentice-Hall, Englewood Cliffs, N. J., 1969.

4. K. de Leeuw, A type of convexity in the space of $n$ complex variables, Trans. Amer. Math. Soc. 83 (1956), 193-204. MR 18, 294.

5. - Functions on circular subsets of the space of $n$ complex variables, Duke Math. J. 24 (1957), 415-431. MR 20 \#1235.

6. H. Rossi, The local maximum modulus principle, Ann. of Math. (2) 72 (1960), 1-11. MR $22 \# 8317$.

7. J. Wermer, On an example of Stolzenberg, Sympos. Several Complex Variables (Park City, Utah, 1970), Lecture Notes in Math., no. 184, Springer-Verlag, Berlin and New York, 1971, pp. 79-84.

8. G. Stolzenberg, A hull with no analytic structure, J. Math. Mech. 12 (1963), 103111. MR $26 \# 627$.

9. A Browder, Introduction to function algebras, Benjamin, New York, 1969. MR 39 $\# 7431$.

10. B. J. Cole, One-point parts and the peak point conjecture, Ph.D. Dissertation, Yale University, New Haven, Conn., 1968.

11. R. McKissick, A non-trivial normal sup norm algebra, Bull. Amer. Math. Soc. 69 (1963), 391-395. MR $26 \# 4166$.

12. A Huber, $\ddot{U} b e r$ Potentiale, welche auf vorgegebenen Mengen verschwinden, Comment. Math. Helv. 43 (1968), 41-50. MR 37 \#50. 
13. A Browder, Point derivations on function algebras, J. Functional Analysis 1 (1967), 22-27. MR $35 \# 2144$.

14. M. S. Mel 'nikov, On the structure of Gleason parts of the algebra $R(E)$, Funkcional. Anal. i Priložen. 1 (1967), 97-100. (Russian) MR 35 \#740.

15. W. Fleming, Functions of several variables, Addison-Wesley, Reading, Mass., 1965. MR 30 \#4875.

16. M. Freeman, Some conditions on uniform approximation on a manifold, Function Algebras, Proc. Internat. Sympos. on Function Algebras (Tulane University, 1965), Scott, Foresman, Chicago, Ill., 1965, pp. 42-60. MR $33 \# 1758$.

DEPAR TMENT OF MATHEMATICS, BROWN UNIVERSITY, PROVIDENCE, RHODE ISLAND 02912

Current address: Department of Mathematics, Yale University, New Haven, Connecticut 06520 